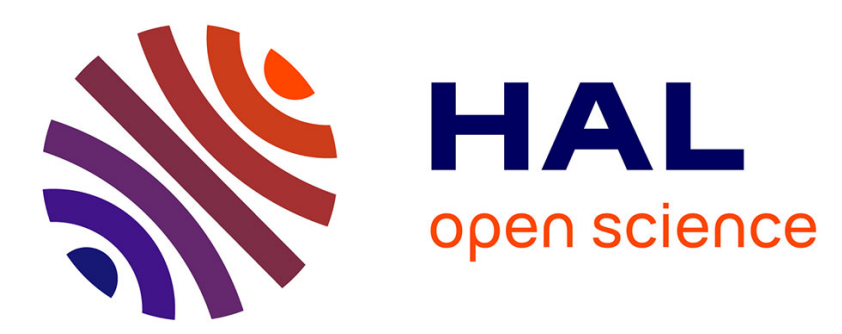

\title{
From trisections in module categories to quasi-directed components
}

\author{
Edson R. Alvares, Ibrahim Assem, Sonia Trepode, Flávio U. Coelho, Maria \\ Ines Peña
}

\section{- To cite this version:}

Edson R. Alvares, Ibrahim Assem, Sonia Trepode, Flávio U. Coelho, Maria Ines Peña. From trisections in module categories to quasi-directed components. 2010. hal-00584591

\section{HAL Id: hal-00584591 \\ https://hal.science/hal-00584591}

Preprint submitted on 8 Apr 2011

HAL is a multi-disciplinary open access archive for the deposit and dissemination of scientific research documents, whether they are published or not. The documents may come from teaching and research institutions in France or abroad, or from public or private research centers.
L'archive ouverte pluridisciplinaire HAL, est destinée au dépôt et à la diffusion de documents scientifiques de niveau recherche, publiés ou non, émanant des établissements d'enseignement et de recherche français ou étrangers, des laboratoires publics ou privés. 


\title{
FROM TRISECTIONS IN MODULE CATEGORIES TO QUASI-DIRECTED COMPONENTS
}

\author{
E. R. ALVARES, I. ASSEM, F. U. COELHO, M. I. PEÑA, AND S. TREPODE
}

\begin{abstract}
In this paper, we define and study a special type of trisections in a module category, namely the compact trisections which characterise quasidirected components. We apply this notion to the study of laura algebras and we use it to define a class of algebras with predictable Auslander-Reiten components.
\end{abstract}

Let $\Lambda$ be an artin algebra, $\bmod \Lambda$ be the category of finitely generated right $\Lambda$-modules and $\operatorname{ind} \Lambda$ be a full subcategory of $\bmod \Lambda$ consisting of exactly one representative from each isomorphism class of indecomposable $\Lambda$-modules. In this paper, we are interested in the study of a particular type of components of the Auslander-Reiten quiver $\Gamma(\bmod \Lambda)$ of $\Lambda$, namely the so-called quasi-directed components which appeared naturally in the study of laura algebras [3, 19]. They are components containing at most finitely many modules lying on oriented cycles and which moreover are generalised standard (in the sense of [23]). These components and their connection to laura algebras have been studied in [27, 14]. Our objective in this paper is to give simple new categorical characterisations of quasi-directed components.

For this purpose, our main tool is the notion of trisection, introduced in [13]: a trisection $(\mathcal{A}, \mathcal{B}, \mathcal{C})$ of ind $\Lambda$ is a triple of full disjoint subcategories of ind $\Lambda$ such that ind $\Lambda=\mathcal{A} \cup \mathcal{B} \cup \mathcal{C}$ and $\operatorname{Hom}_{\Lambda}(\mathcal{B}, \mathcal{A})=\operatorname{Hom}_{\Lambda}(\mathcal{C}, \mathcal{B})=\operatorname{Hom}_{\Lambda}(\mathcal{C}, \mathcal{A})=0$. Here, we define and study a special type of trisections, namely the compact trisections which characterise quasi-directed components. A second approach is motivated by the observation that a trisection $(\mathcal{A}, \mathcal{B}, \mathcal{C})$ in ind $\Lambda$ is really determined by the central subcategory $\mathcal{B}$. Along this line, and in the spirit of [19], we define the core of a component $\Gamma$ to be a full subcategory of ind $\Lambda$ which is the convex envelope in ind $\Lambda$ of a left and of a right section of $\Gamma$ (in the sense of [1]). We prove here that the presence of a core characterises quasi-directed components. Moreover, these conditions yield a description of the module category of $\Lambda$ which then resembles closely that of laura algebra. Namely, our main result is as follows.

THEOREM. The following conditions are equivalent for an artin algebra $\Lambda$ :

(a) $\Gamma(\bmod \Lambda)$ admits a separating quasi-directed component $\Gamma$.

1991 Mathematics Subject Classification. 16G20, 16G70.

Key words and phrases. trisections, cores, quasi-directed components, laura algebras. 
(b) ind $\Lambda$ admits a compact trisection $(\mathcal{A}, \mathcal{B}, \mathcal{C})$.

(c) $\Gamma(\bmod \Lambda)$ admits a separating convex component $\Gamma$ having a left section $\Sigma_{l}$ and a right section $\Sigma_{r}$ whose convex envelope $\Delta$ is a core in $\Gamma$.

If these conditions are satisfied, then $\Gamma$ has only finitely many $\tau_{\Lambda}$-orbits and is convex in $\Gamma(\bmod \Lambda)$. Moreover, if $\Gamma^{\prime} \neq \Gamma$ is a component of $\Gamma(\bmod \Lambda)$, then either $\Gamma^{\prime} \subset \mathcal{A}$ or $\Gamma^{\prime} \subset \mathcal{C}$. Moreover

(a) $\operatorname{Hom}_{\Lambda}\left(\Gamma^{\prime}, \Gamma\right) \neq 0$ implies $\Gamma^{\prime} \subset \mathcal{A}$;

(b) $\operatorname{Hom}_{\Lambda}\left(\Gamma, \Gamma^{\prime}\right) \neq 0$ implies $\Gamma^{\prime} \subset \mathcal{C}$.

In particular, if $\Gamma$ is non-semiregular, then $\Lambda / \operatorname{Ann}(\Gamma)$ is a laura algebra having $\Gamma$ as a unique quasi-directed faithful component while, if $\Gamma$ is semiregular, then $\Lambda / \operatorname{Ann}(\Gamma)$ is a tilted algebra having $\Gamma$ as a connecting component.

Since quasi-directed components are intimately connected to laura algebras, we get, as corollaries, several new characterisations of classes of laura algebras by means of trisections and cores. We end this paper with the construction of a generalisation of the class of laura algebras, which we call quasi-laura, and the $n^{\text {th }}$ iterates of this construction, which we call $n$-quasi-laura.

\section{Preliminaries}

1.1. Notation. Throughout this paper, all our algebras are basic and connected artin algebras. For an algebra $\Lambda$, we denote by $\bmod \Lambda$ its category of finitely generated right $\Lambda$-modules and by ind $\Lambda$ a full subcategory of $\bmod \Lambda$ consisting of exactly one representative from each isomorphism class of indecomposable modules. When we speak about a $\Lambda$-module (or an indecomposable $\Lambda$-module), we always mean implicitly that it belongs to $\bmod \Lambda$ (or to $\operatorname{ind} \Lambda$, respectively). Also, all subcategories of $\bmod \Lambda$ are full and so are identified with their object classes.

A subcategory $\mathcal{C}$ of ind $\Lambda$ is called finite if it has only finitely many objects, and it is cofinite if its complement $\mathcal{C}^{c}=\operatorname{ind} \Lambda \backslash \mathcal{C}$ is finite. We sometimes write $M \in \mathcal{C}$ to express that $M$ is an object in $\mathcal{C}$. We denote by add $\mathcal{C}$ the subcategory of $\bmod \Lambda$ with objects the finite direct sums of summands of modules in $\mathcal{C}$ and, if $M$ is a module, we abbreviate $\operatorname{add}\{M\}$ as add $M$. Similarly, we denote by ind $M$ the subcategory of ind $\Lambda$ having as objects the indecomposable summands of $M$. We denote the projective (or injective) dimension of a module $M$ as $\operatorname{pd} M$ (or $\operatorname{id} M$, respectively). The global dimension of $\Lambda$ is denoted by gl.dim $\Lambda$. For a point $x$ in the quiver of $\Lambda$, we denote by $P_{x}$ (or $I_{x}$, or $S_{x}$ ) the indecomposable projective (or injective, or simple, respectively) module corresponding to this point. Finally, if $\mathcal{C}, \mathcal{D}$ are two full subcategories of $\bmod \Lambda$, we write $\operatorname{Hom}_{\Lambda}(\mathcal{C}, \mathcal{D})=0$ to express that $\operatorname{Hom}_{\Lambda}(X, Y)=0$ for any $X \in \mathcal{C}$ and $Y \in \mathcal{D}$.

For an algebra $\Lambda$, we denote by $\Gamma(\bmod \Lambda)$ its Auslander-Reiten quiver and by $\tau_{\Lambda}=\mathrm{DTr}, \tau_{\Lambda}^{-1}=\operatorname{TrD}$ its Auslander-Reiten translations. For further definitions or facts needed on $\bmod \Lambda$ or $\Gamma(\bmod \Lambda)$, we refer the reader to $[7,8]$. 
1.2. Paths. Let $\Lambda$ be an algebra. Given $M, N \in \operatorname{ind} \Lambda$, a path from $M$ to $N$ in $\operatorname{ind} \Lambda$ (denoted by $M \leadsto N$ ) is a sequence of non-zero morphisms

$$
M=X_{0} \stackrel{f_{1}}{\rightarrow} X_{1} \rightarrow \cdots \rightarrow X_{t-1} \stackrel{f_{t}}{\rightarrow} X_{t}=N
$$

$(t \geq 1)$, where $X_{i} \in \operatorname{ind} \Lambda$ for all $i$. We then say that $M$ is a predecessor of $N$ and $N$ is a successor of $M$. A path from $M$ to $M$ involving at least one nonisomorphism is a cycle. A module $M \in$ ind $\Lambda$ which lies on no cycle is directed. If each $f_{i}$ in $(*)$ is irreducible, we say that $(*)$ is a path of irreducible morphisms. A path $(*)$ of irreducible morphisms is sectional if $\tau_{\Lambda} X_{i+1} \neq X_{i-1}$ for all $i$ with $0<i<t$. A refinement of $(*)$ is a path in ind $\Lambda$

$$
M=X_{0}^{\prime} \longrightarrow X_{1}^{\prime} \longrightarrow \cdots \longrightarrow X_{s-1}^{\prime} \longrightarrow X_{s}^{\prime}=N
$$

such that there is an order-preserving injection $\sigma:\{1, \cdots, t-1\} \rightarrow\{1, \cdots, s-1\}$ satisfying $X_{i}=X_{\sigma i}^{\prime}$ for all $i$ with $0<i<t$.

Let $\mathcal{C}$ be a subcategory of ind $\Lambda$. Then, $\mathcal{C}$ is called directed (or almost directed) if all (or all but finitely many, respectively) objects of $\mathcal{C}$ are directed. Also, $\mathcal{C}$ is closed under predecessors if, whenever $M \leadsto N$ is a path in indC, with $N \in \mathcal{C}$, then $M \in \mathcal{C}$. An example is the left part $\mathcal{L}_{\Lambda}$ of $\bmod \Lambda$, defined in [13], which is the full subcategory of ind $\Lambda$ with object class

$$
\mathcal{L}_{\Lambda}=\{M \in \operatorname{ind} \Lambda: \operatorname{pd} L \leq 1 \text { whenever there is a path } L \leadsto M\}
$$

Another example is, for a given module $M$, the full subcategory $\operatorname{Pred} M$ of the predecessors of $M$ : this is the full subcategory of ind $\Lambda$ consisting of the modules $X$ such that there exist an indecomposable summand $Y$ of $M$ and a path $X \leadsto Y$.

We define dually subcategories closed under successors, examples of which are the right part $\mathcal{R}_{\Lambda}$ of $\bmod \Lambda$, and the subcategory $\operatorname{Succ} M$ of ind $\Lambda$ consisting of the successors of $M$.

Given $X, Y \in \operatorname{ind} \Lambda$, we denote by $\operatorname{rad}_{\Lambda}(X, Y)$ the group of the morphisms $f: X \longrightarrow Y$ which are not isomorphisms and by $\operatorname{rad}_{\Lambda}^{\infty}(X, Y)$ the intersection of all powers $\operatorname{rad}_{\Lambda}^{i}(X, Y), i \geq 1$, of $\operatorname{rad}_{\Lambda}(X, Y)$. The following result has been proven in different versions (see, for instance, $[21](2.1)$ or $[22](2.1)$ ). The one below is $[27](1.1)$.

Lemma. Let $\Lambda$ be an algebra and $X, Y \in$ ind $\Lambda$ be such that $\operatorname{rad}_{\Lambda}^{\infty}(X, Y) \neq 0$. Then, for each $t \geq 0$, there exists a path in ind $\Lambda$

$$
X=X_{0} \stackrel{f_{1}}{\rightarrow} X_{1} \rightarrow \cdots \stackrel{f_{t}}{\rightarrow} X_{t} \stackrel{g_{t}}{\rightarrow} Y
$$

with the $X_{i}$ pairwise non-isomorphic, $g_{t} f_{t} \cdots f_{1} \neq 0$, each $f_{i}$ is a composite of irreducible morphisms and $g_{t} \in \operatorname{rad}_{\Lambda}^{\infty}\left(X_{t}, Y\right)$.

1.3. Covariant and contravariant finiteness. Let $\mathcal{X}$ be an additive subcategory of $\bmod \Lambda$. For a $\Lambda$-module $M$, a right $\mathcal{X}$-approximation of $M$ is a morphism $f_{M}: X_{M} \longrightarrow M$ with $X_{M} \in \mathcal{X}$ such that any morphism $f: X \longrightarrow M$ with $X \in \mathcal{X}$ factors through $f_{M}$. The subcategory $\mathcal{X}$ is contravariantly finite if any 
$\Lambda$-module has a right $\mathcal{X}$-approximation. We define dually left $\mathcal{X}$-approximations and covariantly finite subcategories. Finally, $\mathcal{X}$ is functorially finite if it is both contravariantly and covariantly finite. Observe that finite and cofinite subcategories are functorially finite (see [9]).

1.4. If $\mathcal{X}$ is an additive subcategory of $\bmod \Lambda$, closed under extensions, then a module $M \in \mathcal{X}$ is called Ext-projective (or Ext-injective) in $\mathcal{X}$ if $\left.\operatorname{Ext}_{\Lambda}^{1}(M,-)\right|_{\mathcal{X}}=$ 0 (or $\left.\operatorname{Ext}_{\Lambda}^{1}(-, M)\right|_{\mathcal{X}}=0$, respectively), see [10]. It is shown in [10](3.3)(3.7) that, if $\mathcal{X}$ is a torsion (or torsion-free) class, then an indecomposable module $M \in \mathcal{X}$ is Ext-projective in $\mathcal{X}$ if and only if $\tau_{\Lambda} M$ is torsion-free (or, $M \in \mathcal{X}$ is Ext-injective in $\mathcal{X}$ if and only if $\tau_{\Lambda}^{-1} M$ is torsion).

1.5. We recall from [13] that an algebra $\Lambda$ is called quasi-tilted if gl.dim $\Lambda \leq$ 2 and ind $A=\mathcal{L}_{\Lambda} \cup \mathcal{R}_{\Lambda}$. The module category of a quasi-tilted algebra has been described in $[11,15,16]$. Several generalisations of this notion have been introduced over the years (see [4] for a survey). We recall the definition of laura algebras [3]: an algebra $\Lambda$ is laura if $\mathcal{L}_{\Lambda} \cup \mathcal{R}_{\Lambda}$ is cofinite in ind $\Lambda$.

Quasi-tilted algebras are clearly laura, and a laura algebra which is not quasitilted is called strict. The module category of a strict laura algebra has been described in $[3,19]$. In particular, a strict laura algebra has a unique faithful, non-semiregular Auslander-Reiten component which is quasi-directed (that is, it is generalised standard and almost directed). This component is called its connecting component, in analogy with the connecting components of tilted algebras.

\section{TRISECTIONS}

2.1. Following [13], we define a trisection of ind $\Lambda$.

Definition. Let $\Lambda$ be an artin algebra. A trisection of ind $\Lambda$ is a triple of disjoint full subcategories $(\mathcal{A}, \mathcal{B}, \mathcal{C})$ of ind $\Lambda$ such that:

(a) ind $\Lambda=\mathcal{A} \cup \mathcal{B} \cup \mathcal{C}$, and

(b) $\operatorname{Hom}_{\Lambda}(\mathcal{C}, \mathcal{B})=\operatorname{Hom}_{\Lambda}(\mathcal{C}, \mathcal{A})=\operatorname{Hom}_{\Lambda}(\mathcal{B}, \mathcal{A})=0$.

Notice that this definition of trisection is different from the one given in [18]. If any of $\mathcal{A}, \mathcal{B}, \mathcal{C}$ is empty, then the definition above reduces to that of a split torsion pair.

If $(\mathcal{A}, \mathcal{B}, \mathcal{C})$ is a trisection of $\operatorname{ind} \Lambda$, then the subcategory $\mathcal{A}$ is closed under predecessors and, dually, $\mathcal{C}$ is closed under successors. Also, $\mathcal{B}$ is convex in ind $\Lambda$, that is, whenever there exists a path

$$
X=X_{0} \rightarrow X_{1} \rightarrow \cdots \rightarrow X_{t}=Y
$$

in $\operatorname{ind} \Lambda$, with $X, Y \in \mathcal{B}$, then $X_{i} \in \mathcal{B}$ for each $i$. 
2.2. We need another definition due to Ringel $[20]$ (p. 120). Let $\mathcal{A}, \mathcal{B}, \mathcal{C}$ be three disjoint full subcategories of ind $\Lambda$, then we say that $\mathcal{B}$ separates $\mathcal{A}$ from $\mathcal{C}$ if $\mathcal{B} \neq \emptyset$ and any morphism $X \rightarrow Y$ with $X \in \mathcal{A}$ and $Y \in \mathcal{C}$ factors through add $\mathcal{B}$. If $(\mathcal{A}, \mathcal{B}, \mathcal{C})$ is a trisection and $\mathcal{B}$ separates $\mathcal{A}$ from $\mathcal{C}$, then we say that $(\mathcal{A}, \mathcal{B}, \mathcal{C})$ is a separated trisection.

Lemma. Let $(\mathcal{A}, \mathcal{B}, \mathcal{C})$ be a separated trisection of ind $\Lambda$ and $f: M \rightarrow N$ be an irreducible morphism with $M, N$ indecomposable.

(a) If $M \in \mathcal{A}, N \notin \mathcal{A}$ then $N \in \mathcal{B}$.

(b) If $M \notin \mathcal{C}, N \in \mathcal{C}$ then $M \in \mathcal{B}$.

Proof. We prove only (a) since the proof of (b) is dual.

(a) Assume $M \in \mathcal{A}$ and $N \in \mathcal{C}$, then $f: M \rightarrow N$ must factor through add $\mathcal{B}$. But then $M \in \mathcal{B}$ or else $N \in \mathcal{B}$, a contradiction in either case.

2.3. ExAmples. (a) Let $\Lambda$ be a representation-infinite hereditary algebra, then ind $\Lambda$ admits a separated trisection $(\mathcal{A}, \mathcal{B}, \mathcal{C})$, where $\mathcal{A}$ consists of the postprojective modules, $\mathcal{C}$ of the preinjective, and $\mathcal{B}$ of the regular.

(b) The motivating example is from the study of quasi-tilted algebras: it is shown in [13] (II,1.6) and (II, 1.7) that any quasi-tilted algebra $\Lambda$ admits a trisection $\left(\mathcal{L}_{\Lambda} \backslash \mathcal{R}_{\Lambda}, \mathcal{L}_{\Lambda} \cap \mathcal{R}_{\Lambda}, \mathcal{R}_{\Lambda} \backslash \mathcal{L}_{\Lambda}\right)$. Moreover, by [11] and [15](4.3), this trisection is separated.

(c) Let $\Lambda$ be the radical square zero algebra given by the quiver

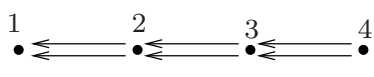

Figure 1.

Then the Auslander-Reiten quiver $\Gamma(\bmod \Lambda)$ of $\Lambda$ is of the form

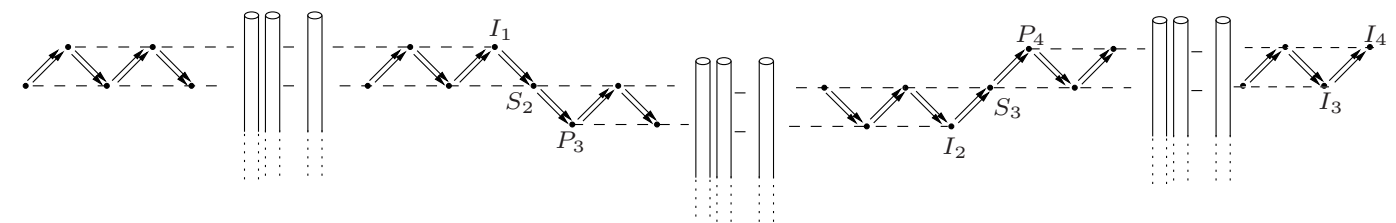

FIGURE 2.

Then ind $\Lambda$ admits a trisection $(\mathcal{A}, \mathcal{B}, \mathcal{C})$ where $\mathcal{A}$ consists of all predecessors of $S_{2}, \mathcal{C}$ of all successors of $S_{3}$ and $\mathcal{B}$ consists of the remaining indecomposables. Trivially, $\mathcal{B}$ separates $\mathcal{A}$ from $\mathcal{C}$. 
2.4. Lemma. The following statements are equivalent for a trisection $(\mathcal{A}, \mathcal{B}, \mathcal{C})$ of ind $\Lambda$.

(a) $p d M \leq 1$ for all $M \in \mathcal{A}$.

(b) $\mathcal{A} \subset \mathcal{L}_{\Lambda}$.

(c) Each indecomposable injective $I \in \mathcal{A}$ satisfies the following: any path $I \leadsto X$ with $X \in \mathcal{A}$ is refinable to a path of irreducible morphisms and any such path is sectional.

Proof. Since $\mathcal{A}$ is closed under predecessors and $\operatorname{pd} M \leq 1$ for each $M \in \mathcal{L}_{\Lambda}$, the equivalence of (a) and (b) is trivial. The equivalence between (b) and (c) follows easily from $[3](1.6)$.

2.5. We need the following lemma, essentially due to Smalø [26].

Lemma. Let $\mathcal{A}$ be a full subcategory of ind $\Lambda$ closed under predecessors. Then add $\mathcal{A}$ is contravariantly finite if and only if add $\mathcal{A}^{c}$ is covariantly finite.

2.6. The main result of this section is the following theorem.

TheOREM. Let $\mathcal{A}, \mathcal{B}, \mathcal{C}$ be three disjoint full subcategories of ind $\Lambda$ such that ind $\Lambda=\mathcal{A} \cup \mathcal{B} \cup \mathcal{C}$ and assume that $\mathcal{B}$ separates $\mathcal{A}$ from $\mathcal{C}$. Then $(\mathcal{A}, \mathcal{B}, \mathcal{C})$ is a separated trisection with addB functorially finite if and only if the following two conditions hold:

(a) add $\mathcal{A}$ is contravariantly finite and closed under predecessors, and

(b) addC is covariantly finite and closed under successors.

Proof. Necessity. We claim that $\operatorname{add}(\mathcal{A} \cup \mathcal{B})$ is contravariantly finite. Let $X \in$ $\operatorname{ind} \Lambda$. If $X \in \mathcal{A} \cup \mathcal{B}$, then the identity $1_{X}: X \rightarrow X$ is a $\operatorname{right} \operatorname{add}(\mathcal{A} \cup \mathcal{B})$ approximation. If $X \notin \mathcal{A} \cup \mathcal{B}$, then $X \in \mathcal{C}$. Since add $\mathcal{B}$ is contravariantly finite, then $X$ has a right add $\mathcal{B}$-approximation $f_{\mathcal{B}}: X_{\mathcal{B}} \rightarrow X$ with $X_{\mathcal{B}} \in \operatorname{add} \mathcal{B} \subset$ $\operatorname{add}(\mathcal{A} \cup \mathcal{B})$. Let $g: Y \rightarrow X$ be a morphism, with $Y \in \operatorname{add}(\mathcal{A} \cup \mathcal{B})$. We may assume that $Y$ is indecomposable. If $Y \in \mathcal{B}$, then $g$ factors through $f_{\mathcal{B}}$. Suppose that $Y \in \mathcal{A}$. Since $\mathcal{B}$ separates $\mathcal{A}$ from $\mathcal{C}$, there exists $Z \in$ add $\mathcal{B}$ and morphisms $g_{2}: Y \rightarrow Z$ and $g_{1}: Z \rightarrow X$ such that $g=g_{1} g_{2}$. Since $g_{1}$ factors through $f_{\mathcal{B}}$, we have established our claim. Applying now (2.5) yields that add $\mathcal{C}$ is covariantly finite. Dually, add $\mathcal{A}$ is contravariantly finite. The other statements in (a),(b) are evident.

Sufficiency. Since $\mathcal{A}$ is closed under predecessors, $\mathcal{C}$ is closed under successors and the subcategories $\mathcal{A}, \mathcal{B}, \mathcal{C}$ are disjoint, we easily infer that

$$
\operatorname{Hom}_{\Lambda}(\mathcal{C}, \mathcal{B})=\operatorname{Hom}_{\Lambda}(\mathcal{C}, \mathcal{A})=\operatorname{Hom}_{\Lambda}(\mathcal{B}, \mathcal{A})=0
$$

and so $(\mathcal{A}, \mathcal{B}, \mathcal{C})$ is a trisection of ind $\Lambda$. It remains to show that add $\mathcal{B}$ is functorially finite.

It follows from the hypothesis that $\mathcal{A} \cup \mathcal{B}$ is closed under predecessors. Since add $\mathcal{C}$ is covariantly finite, then $\operatorname{add}(\mathcal{A} \cup \mathcal{B})$ is contravariantly finite by Lemma (2.5). Let $X \in \operatorname{ind} \Lambda$, then there exists a right $\operatorname{add}(\mathcal{A} \cup \mathcal{B})$-approximation $f_{\mathcal{A} \cup \mathcal{B}}: X_{\mathcal{A} \cup \mathcal{B}} \rightarrow X$ 
with $X_{\mathcal{A} \cup \mathcal{B}} \in \operatorname{add}(\mathcal{A} \cup \mathcal{B})$. Write $X_{\mathcal{A} \cup \mathcal{B}}=X_{\mathcal{A}} \oplus X_{\mathcal{B}}$ with $X_{\mathcal{A}} \in \operatorname{add} \mathcal{A}$ and $X_{\mathcal{B}} \in$ add $\mathcal{B}$. Let $\iota: X_{\mathcal{B}} \rightarrow X_{\mathcal{A} \cup \mathcal{B}}$ be the natural inclusion and define $f_{\mathcal{B}}: X_{\mathcal{B}} \rightarrow X$ to be the composition $f_{\mathcal{A} \cup \mathcal{B}} \circ \iota$. Let $g: Y \rightarrow X$ be a morphism with $Y \in \operatorname{add} \mathcal{B}$. Since $Y \in \operatorname{add}(\mathcal{A} \cup \mathcal{B})$, then $g$ factors through $f_{\mathcal{A} \cup \mathcal{B}}$ that is, there exists $h: Y \rightarrow X_{\mathcal{A} \cup \mathcal{B}}$ such that $g=f_{\mathcal{A} \cup \mathcal{B}} \circ h$.

Since $Y \in \operatorname{add} \mathcal{B}$, then $\operatorname{Hom}_{\Lambda}\left(Y, X_{\mathcal{A}}\right)=0$. Therefore $\operatorname{Im} h \subset X_{\mathcal{B}}$ and we have the required factorisation by $f_{\mathcal{B}}$. This shows that add $\mathcal{B}$ is contravariantly finite. Dually, add $\mathcal{B}$ is covariantly finite. Hence it is functorially finite.

Note that the conditions of the theorem are satisfied by the algebra $\Lambda$ of Example $(2.3)(\mathrm{c})$, or by any tilted algebra with a non-semiregular connecting component.

\section{Compact Trisections}

3.1. Let $\Lambda$ be an artin algebra. We recall that a subcategory $\mathcal{B}$ of $\operatorname{ind} \Lambda$ is called connected if, for any $X, Y \in \mathcal{B}$, there exists a walk $X=X_{0}-X_{1}-\cdots-X_{t}=Y$ in ind $\Lambda$ with all $X_{i} \in \mathcal{B}$. Here, $M-N$ indicates that there exists either a morphism $M \rightarrow N$ or a morphism $N \rightarrow M$.

Lemma. Let $\Lambda$ be an artin algebra, and $\mathcal{B}$ be a non-empty finite, connected and convex subcategory of ind $\Lambda$. Then there is a unique component $\Gamma^{\mathcal{B}}$ of $\Gamma(\bmod A)$ such that $\Gamma^{\mathcal{B}}$ intersects $\mathcal{B}$.

Proof. If $\Lambda$ is representation-finite, there is nothing to prove. Assume $\Lambda$ to be representation-infinite and suppose there are two modules $M, N \in \mathcal{B}$ and a component $\Gamma \subset \Gamma(\bmod A)$ such that $M \notin \Gamma$ and $N \in \Gamma$. Since $\mathcal{B}$ is connected, then we can suppose, without loss of generality, that there exists a morphism $f: M \longrightarrow N$ or $f: N \longrightarrow M$ in $\bmod \Lambda$. Assume the former. Since $M \notin \Gamma$ and $N \in \Gamma$, then $f \in \operatorname{rad}_{\Lambda}^{\infty}(M, N)$. By (1.2), for each $t \geq 1$, there exists a path

$$
M=M_{0} \stackrel{f_{1}}{\longrightarrow} M_{1} \longrightarrow \cdots \stackrel{f_{t}}{\longrightarrow} M_{t} \stackrel{g_{t}}{\longrightarrow} N
$$

in ind $\Lambda$ such that the $M_{i}$ are pairwise non-isomorphic, $g_{t} f_{t} \cdots f_{1} \neq 0$, each $f_{i}$ is a composite of irreducible morphisms and $g_{t} \in \operatorname{rad}^{\infty}\left(M_{t}, N\right)$. Now, by convexity, we infer that $M_{i} \in \mathcal{B}$, for each $i=1, \cdots, t$, thus getting a contradiction to the finiteness of $\mathcal{B}$. A similar proof holds for a morphism $f: N \longrightarrow M$.

3.2. Lemma. Let $\Lambda$ be an artin algebra, and $(\mathcal{A}, \mathcal{B}, \mathcal{C})$ be a separated trisection of ind $\Lambda$, with $\mathcal{B}$ finite and connected. Let $\Gamma$ be a component of $\Gamma(\bmod \Lambda)$.

(a) If $\Gamma \neq \Gamma^{\mathcal{B}}$, then either $\Gamma \subset \mathcal{A}$ or $\Gamma \subset \mathcal{C}$.

(b) If $\Gamma \cap \mathcal{A} \neq \emptyset$ and $\Gamma \cap \mathcal{C} \neq \emptyset$, then $\Gamma=\Gamma^{\mathcal{B}}$.

Proof. (a) Since $\Gamma \neq \Gamma^{\mathcal{B}}$, clearly any indecomposable module in $\Gamma$ lies either in $\mathcal{A}$ or in $\mathcal{C}$. There remains to show that, if $M, N \in \Gamma$, then both lie in $\mathcal{A}$ or both lie in $\mathcal{C}$. If this is not the case, then we can assume that we have an irreducible morphism $M \rightarrow N$ or $N \rightarrow M$. By definition of trisection, we can supppose that 
we have an irreducible morphism $M \rightarrow N$ with $M \in \mathcal{A}$ and $N \in \mathcal{C}$. But this contradicts (2.2).

(b) Let $M$ be a module in $\mathcal{A}$, and $N$ be a module in $\mathcal{C}$, both lying in $\Gamma$. Then there exists a walk $M=M_{0}-M_{1}-\cdots-M_{t}=N$ (with $t \geq 1$ ) in $\Gamma$. Let $j$ be the least integer such that $M_{j} \in \mathcal{A}$ but $M_{j+1} \notin \mathcal{A}$. Since $\mathcal{A}$ is closed under predecessors, then we have an irreducible morphism $M_{j} \rightarrow M_{j+1}$. But then (2.2) yields $M_{j+1} \in \mathcal{B}$. Therefore $\Gamma=\Gamma^{\mathcal{B}}$ because of (3.1).

3.3. Definition. A trisection $(\mathcal{A}, \mathcal{B}, \mathcal{C})$ is called compact if it is separated and $\mathcal{B}$ is finite, connected and contains all the projectives and all the injectives in $\Gamma^{\mathcal{B}}$.

REMARK. Let $(\mathcal{A}, \mathcal{B}, \mathcal{C})$ be a compact trisection. Since $\mathcal{B}$ is finite, then it is functorially finite and so the results of Section 2 apply trivially. The converse is however not true since the trisection shown in Example 2.3(c) is not compact, but it is functorially finite.

ExAmples. (a) Let $\Lambda$ be an artin algebra. Then, $\Lambda$ is representation-finite if and only if $(\emptyset$,ind $\Lambda, \emptyset)$ is the unique compact trisection of $\operatorname{ind} \Lambda$.

(b) In [21](2.7)p.215, Ringel considered the gluing of two tame hereditary algebras to form a tame one-relation algebra (see also [17]). Since these are tilted algebras, one may define a compact trisection by letting $\mathcal{B}$ be the separating subclass consisting of modules lying on a given complete slice.

(c) Let $\Lambda$ be the radical square zero algebra given by the quiver

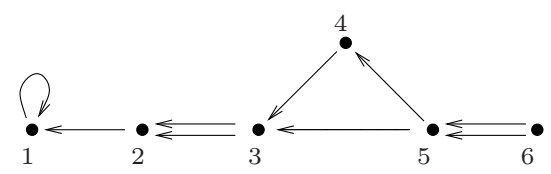

FiguRE 3.

Then $\Gamma(\bmod \Lambda)$ is of the form

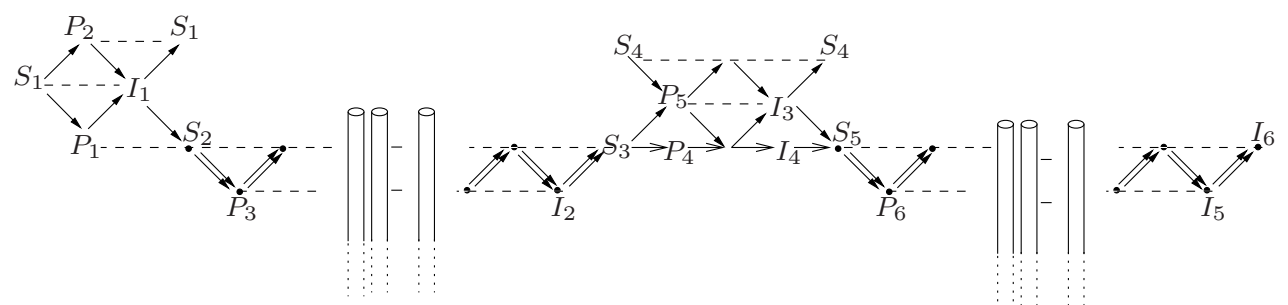

FiguRE 4. 
where we identify the two copies of the simple module $S_{1}$ and the two copies of the simple $S_{4}$. Here, we let $\mathcal{A}$ consist of all predecessors of $\tau S_{3}$ and $\mathcal{C}$ of all successors of $\tau^{-} S_{5}$.

3.4. Let $\Lambda$ be an artin algebra, and let $\Gamma$ be a component of $\Gamma(\bmod \Lambda)$. We recall from [1] that a full subquiver $\Sigma$ of $\Gamma$ is called a right (or left) section provided:

(1) $\Sigma$ is acyclic,

(2) $\Sigma$ is convex in $\Gamma$, and

(3) for each $Y \in \Gamma$ such that there exists a path from $\Sigma$ to $Y$ (or from $Y$ to $\Sigma$, respectively), there exists a unique $s \geq 0$ (or $s \leq 0$, respectively) such that $\tau_{\Lambda}^{s} Y \in \Sigma$.

Lemma. Let $(\mathcal{A}, \mathcal{B}, \mathcal{C})$ be a compact trisection in ind $\Lambda$. Then

(a) The class $\Sigma_{r}$ of the indecomposable Ext-projectives in $\Gamma^{\mathcal{B}} \cap \mathcal{C}$ forms a right section in $\Gamma^{\mathcal{B}}$, and

(b) The class $\Sigma_{l}$ of the indecomposable Ext-injectives in $\Gamma^{\mathcal{B}} \cap \mathcal{A}$ forms a left section in $\Gamma^{\mathcal{B}}$.

Proof. We prove only (a), since the proof of (b) is dual.

(a) The acyclicity of $\Sigma_{r}$ follows from the dual of [1](5.3), and its convexity in $\Gamma^{\mathcal{B}}$ follows from $[6](2.2)$, because $\Gamma^{\mathcal{B}} \cap \mathcal{C}$ contains no projective modules. Let now $X \in \Gamma^{\mathcal{B}}$ be a successor of $\Sigma_{r}$ and consider a walk $Y=Y_{0}-Y_{1}-\cdots-Y_{t}=X$, with $Y \notin \mathcal{C}$ and $Y_{i} \in \mathcal{C}$ for all $i$ such that $1 \leq i \leq t$. Since all the projective modules of $\Gamma^{\mathcal{B}}$ are in $\mathcal{B}$, and applying $\tau_{\Lambda}$ if necessary, the above walk gives rise to a path from $\tau_{\Lambda}^{r} X$, for some $r \geq 0$, to a module not in $\mathcal{C}$. In particular, $\tau_{\Lambda}^{r} X \notin \mathcal{C}$. Hence there exists $s \geq 0$ such that $\tau_{\Lambda}^{s} X \in \mathcal{C}$ but $\tau_{\Lambda}^{s+1} X \notin \mathcal{C}$, implying that $\tau_{\Lambda}^{s} X$ is an Ext-projective of $\Gamma^{\mathcal{B}} \cap \mathcal{C}$. Hence $\tau_{\Lambda}^{s} X \in \Sigma_{r}$, as required.

3.5. Corollary. Let $(\mathcal{A}, \mathcal{B}, \mathcal{C})$ be a compact trisection in ind $\Lambda$. Then all modules in $\Gamma^{\mathcal{B}} \backslash \mathcal{B}$ are directed in $\Gamma^{\mathcal{B}}$.

Proof. If $X \in \Gamma^{\mathcal{B}} \backslash \mathcal{B}$ is not directed in $\Gamma^{\mathcal{B}}$, then there exists a cycle $X=X_{0} \longrightarrow$ $X_{1} \longrightarrow \cdots \longrightarrow X_{t}=X$ between indecomposable $\Lambda$-modules on $\Gamma^{\mathcal{B}}$. Assume first that $X \in \mathcal{C}$. The same argument as in (3.4) yields an $r \geq 0$ such that the path

$$
\tau^{r} X=\tau^{r} X_{0} \longrightarrow \tau^{r} X_{1} \longrightarrow \cdots \longrightarrow \tau^{r} X_{t}=\tau^{r} X
$$

intersects $\Sigma_{r}$, contradicting its acyclicity and its convexity. Similarly if $X \in$ $\mathcal{A}$.

3.6. Corollary. Let $(\mathcal{A}, \mathcal{B}, \mathcal{C})$ be a compact trisection in ind $\Lambda$ and let $X \in \Gamma^{\mathcal{B}}$.

(a) If $X \in \mathcal{C}$, then $X$ has only finitely many predecessors in $\mathcal{C}$.

(b) If $X \in \mathcal{A}$, then $X$ has only finitely many successors in $\mathcal{A}$.

Proof. We only prove (a) since the proof of (b) is dual.

(a) It follows from 2.6 that the subcategory $a d d \mathcal{C}$ is covariantly finite in $\bmod \Lambda$. Because of $[1](8.1)$ every indecomposable module $X \in \Gamma^{\mathcal{B}} \cap \mathcal{C}$ is generated by 
modules in $\Sigma_{r}$ and hence is a successor of $\Sigma_{r}$ in ind $\Lambda$ and from (3.4) we know that $\Sigma_{r}$ is a right section in $\Gamma^{\mathcal{B}} \cap \mathcal{C}$, then if $M \in \Gamma^{\mathcal{B}} \cap \mathcal{C}$ we have that $\Sigma_{r}$ intersects the $\tau_{\Lambda}$-orbit of $M$.

The first consequence of this fact is that $\Sigma_{r}$ intersects each $\tau_{\Lambda}$-orbit of $\Gamma^{\mathcal{B}} \cap \mathcal{C}$ at least once. Since it intersects it at most once [1](5.3)(c), it intersects it exactly once and then the number of $\tau_{\Lambda}$-orbit of $\Gamma^{\mathcal{B}} \cap \mathcal{C}$ equals $\left|\left(\Sigma_{r}\right)_{0}\right| \leq$ rk $K_{0}(\Lambda)$ (this inequality is from $[1](5.3)(\mathrm{b}))$.

The second consequence is that if $M \in \Gamma^{\mathcal{B}} \cap \mathcal{C}$, then there exists a path from $\Sigma_{r}$ to $M$ in $\Gamma^{\mathcal{B}}$, and thus $\Gamma^{\mathcal{B}} \cap \mathcal{C} \subseteq \Gamma_{\geq \Sigma_{r}}$. Now, because of [1] (2.2), $\Gamma_{\geq \Sigma_{r}}$ is isomorphic to a full tranlation subquiver of $\mathbb{N} \Sigma_{r}$, hence each $X \in \Gamma^{\mathcal{B}} \cap \mathcal{C}$ has only finitely many predecessors in $\Gamma^{\mathcal{B}} \cap \mathcal{C}$.

We now observe that the predecessors of $X$ in $\mathcal{C}$ are exactly those lying in $\Gamma^{\mathcal{B}}$. Indeed, if $f: Y \longrightarrow X$ is a non-zero morphism with $Y \in \mathcal{C} \backslash \Gamma^{\mathcal{B}}$, then $f \in$ $\operatorname{rad}_{\Lambda}^{\infty}(Y, X)$. But then (1.2) yields infinitely many predecessors of $X$ lying in $\Gamma^{\mathcal{B}} \cap \mathcal{C}$, a contradiction.

3.7. Lemma. Let $(\mathcal{A}, \mathcal{B}, \mathcal{C})$ be a compact trisection in ind $\Lambda$ and let $M \notin \Gamma^{\mathcal{B}}$ be an indecomposable module.

(a) If $\operatorname{Hom}_{\Lambda}\left(M, \Gamma^{\mathcal{B}}\right) \neq 0$, then $M \in \mathcal{A}$.

(b) If $\operatorname{Hom}_{\Lambda}\left(\Gamma^{\mathcal{B}}, M\right) \neq 0$, then $M \in \mathcal{C}$.

(c) Either $\operatorname{Hom}_{\Lambda}\left(\Gamma^{\mathcal{B}}, M\right)=0$ or $\operatorname{Hom}_{\Lambda}\left(M, \Gamma^{\mathcal{B}}\right)=0$.

Proof. (a) Suppose there is a non-zero morphism $f: M \rightarrow N$ with $N \in \Gamma^{\mathcal{B}}$. Clearly, $f \in \operatorname{rad}_{\Lambda}^{\infty}(M, N)$. So, by the dual of (1.2), there exists, for each $t \geq 1$, a path in ind $\Lambda$

$$
M \stackrel{g_{t}}{\rightarrow} M_{t} \stackrel{f_{t}}{\rightarrow} M_{t-1} \rightarrow \cdots \rightarrow M_{1} \stackrel{f_{1}}{\rightarrow} M_{0}=N
$$

where the $M_{i}$ are pairwise non-isomorphic, $f_{1} \cdots f_{t} g_{t} \neq 0$, each $f_{i}$ is a composition of irreducible morphisms and $g_{t} \in \operatorname{rad}_{\Lambda}^{\infty}\left(M, M_{t}\right)$. If $N \in \mathcal{A}$, then $M \in \mathcal{A}$ because $\mathcal{A}$ is closed under predecessors. If $N \in \mathcal{B}$, then the finiteness of $\mathcal{B}$ yields an $i_{0} \geq 0$ such that $i \geq i_{0}$ implies $M_{i} \in \mathcal{A}$. Again, $M \in \mathcal{A}$. If $N \in \mathcal{C}$ then, by (3.6), $N$ has only a finite number of predecessors in $\mathcal{C}$. Also, $\mathcal{B}$ is finite. Therefore, there exists $i_{0} \geq 0$ such that $i \geq i_{0}$ implies $M_{i} \in \mathcal{A}$, and thus $M \in \mathcal{A}$.

(b) The proof is dual.

(c) It follows from (a) and (b).

3.8. Corollary. Let $(\mathcal{A}, \mathcal{B}, \mathcal{C})$ be a compact trisection in ind $\Lambda$, then $\Gamma^{\mathcal{B}}$ is convex in ind $\Lambda$, and quasi-directed.

Proof. Let $M=M_{0} \longrightarrow M_{1} \longrightarrow \cdots \longrightarrow M_{t-1} \longrightarrow M_{t}=N$ be a path in ind $\Lambda$, with $t \geq 2, M, N \in \Gamma^{\mathcal{B}}$ and $M_{i} \notin \Gamma^{\mathcal{B}}$ for all $i$ such that $1 \leq i<t$. If both $M, N$ are in $\mathcal{B}$, we are done. Thus we may assume that $M$ or $N$ do not lie in $\mathcal{B}$. By (3.7), $M_{1} \in \mathcal{C}$ and $M_{t-1} \in \mathcal{A}$. But $\mathcal{A}$ is closed under predecessors, and $\mathcal{C}$ is closed 
under successors. Hence a contradiction. This proves the convexity.

Because of (3.5), any module in $\Gamma^{\mathcal{B}} \cap \mathcal{A}$ or in $\Gamma^{\mathcal{B}} \cap \mathcal{C}$ is directed in $\Gamma^{\mathcal{B}}$, hence in $\operatorname{ind} \Lambda$ (because of convexity). So, if a module of $\Gamma^{\mathcal{B}}$ is not directed, then it must lie in $\mathcal{B}$. Since $\mathcal{B}$ is finite, $\Gamma^{\mathcal{B}}$ is almost directed. By $[14](4.2 .3), \Gamma^{\mathcal{B}}$ is quasi-directed (and, in particular, generalised standard).

\section{Cores}

4.1. The following definition is inspired by the notion of multisection of [19].

Definition. Let $\Lambda$ be an artin algebra and $\Gamma$ be a component of $\Gamma(\bmod \Lambda)$. A full subcategory $\Delta$ of $\Gamma$ is a core of $\Gamma$ (and $\Gamma$ is said to have a core) if:

(a) $\Delta$ is convex in ind $\Lambda$.

(b) $\Delta$ intersects each $\tau_{\Lambda}$-orbit in $\Gamma$, and only finitely many times.

(c) $\Delta$ is almost directed.

EXAMPLES. (a) A complete slice in the connecting component of a tilted algebra is a core in this component.

(b) Let $\Lambda$ be a representation-infinite strict laura algebra, and $\Gamma$ be a connecting component of $\Gamma(\bmod \Lambda)$. Let $\Sigma_{l}$ be a left section, and $\Sigma_{r}$ be a right section of $\Gamma$. Then the convex hull $\Delta=\mathcal{C}\left(\Sigma_{l} \cup \Sigma_{r}\right)$ of $\Sigma_{l}$ and $\Sigma_{r}$ (that is, the full subcategory consisting of all the modules $M \in \Gamma$ such that there is a path $M^{\prime} \leadsto M \leadsto M^{\prime \prime}$, with $\left.M^{\prime}, M^{\prime \prime} \in \Sigma_{l} \cup \Sigma_{r}\right)$ is a core in $\Gamma$, which contains all the non-directed modules of $\Gamma$. Indeed, by [1](2.2), all modules which are either proper predecessors of $\Sigma_{l}$ (or proper successors of $\Sigma_{r}$ ) in $\Gamma$ are directed. Hence all non-directed modules in $\Gamma$ lie in $\Delta$. Since there are only finitely many of them (because $\Lambda$ is laura), $\Delta$ is almost directed. It follows from the definition of $\Delta$ that it is convex in ind $\Lambda$. Let $X \in \Gamma$ be a proper predecessor of $\Sigma_{l}$ (or a proper successor of $\Sigma_{r}$ ), then clearly its $\tau_{\Lambda}$-orbit intersects $\Sigma_{l}$ (or $\Sigma_{r}$, respectively) exactly once. If $X \in \Gamma$ is neither, then $X \in \Delta$ so its $\tau_{\Lambda}$-orbit intersects $\Delta$. It can only intersect it finitely many times because, by the description of the connecting component of a laura algebra [3], $\Delta$ is finite.

4.2. Before stating, and proving, the main result of this section, we need one last definition.

Definition. Let $\Lambda$ be an artin algebra, a component $\Gamma$ of $\Gamma(\bmod \Lambda)$ is separating if the components distinct from $\Gamma$ belong to one of two classes $\overleftarrow{\Gamma}$ and $\vec{\Gamma}$ such that, if $\Gamma^{\prime} \in \overleftarrow{\Gamma}$ and $\Gamma^{\prime \prime} \in \vec{\Gamma}$, then

(i) $\operatorname{Hom}_{\Lambda}\left(\Gamma, \Gamma^{\prime}\right)=0, \operatorname{Hom}_{\Lambda}\left(\Gamma^{\prime \prime}, \Gamma\right)=0, \operatorname{Hom}_{\Lambda}\left(\Gamma^{\prime \prime}, \Gamma^{\prime}\right)=0$.

(ii) Any morphism $M^{\prime} \rightarrow M^{\prime \prime}$ with $M^{\prime} \in \Gamma^{\prime}$ and $M^{\prime \prime} \in \Gamma^{\prime \prime}$ factors through $\operatorname{add} \Gamma$.

As an easy example, a connecting component of a tilted algebra is separating. 
THEOREM. The following conditions are equivalent for an artin algebra $\Lambda$ :

(a) $\Gamma(\bmod \Lambda)$ admits a separating quasi-directed component $\Gamma$.

(b) ind $\Lambda$ admits a compact trisection $(\mathcal{A}, \mathcal{B}, \mathcal{C})$.

(c) $\Gamma(\bmod \Lambda)$ admits a separating convex component $\Gamma$ having a left section $\Sigma_{l}$ and a right section $\Sigma_{r}$ whose convex envelope $\Delta$ is a core in $\Gamma$.

If these conditions are satisfied, and $\Gamma^{\prime} \neq \Gamma$ is a component of $\Gamma(\bmod \Lambda)$, then either $\Gamma^{\prime} \subset \mathcal{A}$ or $\Gamma^{\prime} \subset \mathcal{C}$. Moreover,

(i) $\operatorname{Hom}_{\Lambda}\left(\Gamma^{\prime}, \Gamma\right) \neq 0$ implies $\Gamma^{\prime} \subset \mathcal{A}$;

(ii) $\operatorname{Hom}_{\Lambda}\left(\Gamma, \Gamma^{\prime}\right) \neq 0$ implies $\Gamma^{\prime} \subset \mathcal{C}$.

Proof. (a) $\Rightarrow$ (b). Assume that $\Lambda$ is an algebra having a separating quasi-directed component $\Gamma$. We may assume that $\Lambda$ is representation-infinite. In particular, the left stable or the right stable part of $\Gamma$ is infinite or both are. Let $\left\{{ }_{i} \Sigma\right\}$ be a family of sections in the connected components of the left stable part ${ }_{l} \Gamma$ and let $\left\{\Sigma_{j}^{\prime}\right\}$ be a family of sections in the connected components of the right stable part $\Gamma_{r}$. Next, we define $\mathcal{B}$ to consist of all the indecomposable modules which are neither proper predecessors of the ${ }_{i} \Sigma$, nor proper successors of the $\Sigma_{j}^{\prime}$ (that is, lying between the ${ }_{i} \Sigma$ and the $\Sigma_{j}^{\prime}$ ). We define $\Gamma \cap \mathcal{A}$ (or $\Gamma \cap \mathcal{C}$ ) to consist of all the indecomposables in $\Gamma$ which are proper predecessors of the ${ }_{i} \Sigma$ (or proper successors of the $\Sigma_{j}^{\prime}$, respectively). Also, if $\Gamma^{\prime} \neq \Gamma$ is a component of $\Gamma(\bmod \Lambda)$, we let $\Gamma^{\prime} \subset \mathcal{A}$ if $\Gamma^{\prime} \in \overleftarrow{\Gamma}$ and $\Gamma^{\prime} \subset \mathcal{C}$ if $\Gamma^{\prime} \in \vec{\Gamma}$. By [27](2.4.1 and 2.4.2) we have that $\mathcal{B}$ is finite. It is then easily seen that $(\mathcal{A}, \mathcal{B}, \mathcal{C})$ is a compact trisection of $\operatorname{ind} \Lambda$.

(b) $\Rightarrow(\mathrm{c})$. By (3.8), the component $\Gamma=\Gamma^{\mathcal{B}}$ is convex (and even quasi-directed). Let $\overleftarrow{\Gamma}^{\mathcal{B}}$ and $\vec{\Gamma}^{\mathcal{B}}$ consist of the components of $\Gamma(\bmod \Lambda)$ distinct from $\Gamma^{\mathcal{B}}$, and lying in $\mathcal{A}$ and $\mathcal{C}$, respectively (this definition makes sense because of (3.2)). It is then evident that $\Gamma^{\mathcal{B}}$ is separating.

By (3.4), the class $\Sigma_{l}$ (or $\Sigma_{r}$ ) of the Ext-injectives in $\Gamma \cap \mathcal{A}$ (or of the Extprojectives in $\Gamma \cap \mathcal{C}$ ) is a left section (or a right section, respectively). Replacing each of $\Sigma_{l}$ and $\Sigma_{r}$, if necessary, by their Auslander-Reiten translates, we may assume that no projective and no injective module in $\Gamma^{\mathcal{B}}$ is a predecessor of $\Sigma_{l}$, or a successor of $\Sigma_{r}$. Since the convex hull $\Delta$ of $\Sigma_{l}$ and $\Sigma_{r}$ is clearly connected and convex in ind $\Lambda$ (because it is convex in the convex component $\Gamma^{\mathcal{B}}$ ), we have to show that $\Delta$ intersects each $\tau_{\Lambda}$-orbit in $\Gamma^{\mathcal{B}}$ finitely many times, and that it is almost directed. Note first that, since $\mathcal{B}$ is a finite subcategory of the quasidirected component $\Gamma^{\mathcal{B}}$, then $\mathcal{B}$ has finitely many $\tau_{\Lambda}$-orbits.

We claim that $\Delta=\Sigma_{l} \cup \mathcal{B} \cup \Sigma_{r}$. This yields the result because: (i) each of $\Sigma_{l}, \mathcal{B}$ and $\Sigma_{r}$ is finite; (ii) $\Sigma_{l}$ crosses each $\tau_{\Lambda}$-orbit of $\Gamma \cap \mathcal{A}$; (iii) $\Sigma_{r}$ crosses each $\tau_{\Lambda}$-orbit of $\Gamma \cap \mathcal{C}$; (iv) all the non-directing modules of $\Gamma$ lie in $\mathcal{B}$ by (3.5). Observe first that $\mathcal{B} \subset \Delta$. Indeed, if $X \in \mathcal{B} \backslash \Delta$, then $X$ precedes $\Sigma_{l}$ or succedes $\Sigma_{r}$. In the first case, $X$ precedes an Ext-injective in $\Gamma \cap \mathcal{A}$. Therefore $X \in \mathcal{A}$. Similarly, in the second case, $X \in \mathcal{C}$ and we are done. So $\Sigma_{l} \cup \mathcal{B} \cup \Sigma_{r} \subset \Delta$. 
Now, if $X \in \Delta \backslash\left(\Sigma_{l} \cup \Sigma_{r}\right)$, then there exists a path $E^{\prime} \leadsto X \leadsto U^{\prime}$ of irreducible morphisms, with $E^{\prime} \in \Sigma_{l}$ and $U^{\prime} \in \Sigma_{r}$. We may assume that the immediate successor of $E^{\prime}$ on this path in not is $\Sigma_{l}$. By $(2.2)$, it lies in $\mathcal{B}$. Similarly for the immediate predecessor of $U^{\prime}$. But this implies that $X \in \mathcal{B}$ and the claim is proven.

(c) $\Rightarrow$ (a) Let $\mathcal{A}$ consist of the predecessors of $\Sigma_{l}, \mathcal{B}=\Delta \backslash\left(\Sigma_{l} \cup \Sigma_{r}\right)$ and $\mathcal{C}$ consist of the successors of $\Sigma_{r}$. Clearly, $\Gamma^{\mathcal{B}}=\Gamma$. By hypothesis, $\Gamma$ is convex and almost directed. By [14](4.2.3), it is quasi-directed. This proves (a)

The last statement follows from (3.2).

As a consequence, we have a description of the Auslander-Reiten components of $\Gamma(\bmod \Lambda)$ where $\Lambda$ admits a compact trisection $(\mathcal{A}, \mathcal{B}, \mathcal{C})$. There is, first, the separating quasi-directed convex component $\Gamma^{\mathcal{B}}$, and the other components belong either to $\mathcal{A}$ or to $\mathcal{C}$ (according as to whether they precede, or succede, $\Gamma^{\mathcal{B}}$ ). Let $A=\operatorname{End}\left(\bigoplus_{P_{x} \in \mathcal{A}} P_{x}\right)$ be the support algebra of $\mathcal{A}$ (see [1]), then any component of $\Gamma(\bmod \Lambda)$ which lies in $\mathcal{A}$ is also a component of $\Gamma(\bmod A)$. Dually, a component of $\Gamma(\bmod \Lambda)$ which lies in $\mathcal{C}$ is also an Auslander-Reiten component of the support algebra of $\mathcal{C}$.

4.3. Corollary. Let $\Lambda$ be an algebra admitting a compact trisection $(\mathcal{A}, \mathcal{B}, \mathcal{C})$. Let $\Lambda_{\mathcal{B}}=$ End $P$, where $P$ is the direct sum of all indecomposable projective $\Lambda$-modules $P_{x}$ such that $P_{x}$ and $I_{x}$ both lie in $\mathcal{B}$. Then

(a) ind $\Lambda_{\mathcal{B}} \subset \mathcal{B}$ and so $\Lambda_{\mathcal{B}}$ is representation-finite.

(b) $\Lambda_{\mathcal{B}}$ is a full convex subcategory of $\Lambda$.

Proof. (a) Let $M$ be an indecomposable $\Lambda_{\mathcal{B}}$-module and $e_{x} \in \Lambda_{\mathcal{B}}$ be a primitive idempotent such that $M e_{x} \neq 0$. Then there exist non-zero morphisms $P_{x} \rightarrow$ $M \rightarrow I_{x}$. Since $P_{x}, I_{x} \in \mathcal{B}$, the convexity of $\mathcal{B}$ gives $M \in \mathcal{B}$. Hence $\operatorname{ind} \Lambda_{\mathcal{B}} \subset \mathcal{B}$.

(b) Let $x=x_{0} \rightarrow x_{1} \rightarrow \cdots \rightarrow x_{t}=y$ be a path in the quiver of $\Lambda$, with $x, y$ in the quiver of $\Lambda_{\mathcal{B}}$. Then we have a path of projectives

$$
P_{y}=P_{x_{t}} \rightarrow \cdots \rightarrow P_{x_{1}} \rightarrow P_{x_{0}}=P_{x} .
$$

Since $P_{y}, P_{x} \in \mathcal{B}$, then $P_{x_{i}} \in \mathcal{B}$ for every $i$. Similarly, $I_{x_{i}} \in \mathcal{B}$ for every $i$. Therefore $x_{i}$ belongs to the quiver of $\Lambda_{\mathcal{B}}$.

4.4. Let $\Lambda$ admit a compact trisection $(\mathcal{A}, \mathcal{B}, \mathcal{C})$, the component $\Gamma^{\mathcal{B}}$ is not in general non-semiregular, in contrast with the case of the strict laura algebras. Let indeed $\Lambda$ be the endomorphism algebra of a regular tilting module over the path algebra of a (wild) quiver $Q$, and $\mathcal{B}$ consist of a complete slice, then $\Gamma^{\mathcal{B}}$ is of the form $\mathbb{Z} Q$.

Proposition. Let $\Lambda$ be an algebra admitting a compact trisection $(\mathcal{A}, \mathcal{B}, \mathcal{C})$, then we have one of two cases: 
(a) either $\Gamma^{\mathcal{B}}$ is non-semiregular, in which case the algebra $\Lambda / A n n \Gamma^{\mathcal{B}}$ is a laura algebra;

(b) or $\Gamma^{\mathcal{B}}$ is the connecting component of the algebra $\Lambda / A n n \Gamma^{\mathcal{B}}$ which is tilted.

Proof. Let $\Lambda^{\prime}=\Lambda / \operatorname{Ann} \Gamma^{\mathcal{B}}$. Then $\Gamma^{\mathcal{B}}$ is a component of $\Gamma\left(\bmod \Lambda^{\prime}\right)$. It is faithful, because of the definition of $\Lambda^{\prime}$, and generalised standard, by [23] (Lemma $2)$. Therefore, it is quasi-directed as a component of $\Gamma\left(\bmod \Lambda^{\prime}\right)$. If it is not non-semiregular, then either it is regular, or it is semiregular. In the first case, it follows from [24] that it is of the form $\mathbb{Z} Q$ (for some wild quiver $Q$ ), and is the connecting component of $\Gamma\left(\bmod \Lambda^{\prime}\right)$. In the second case, it follows from [27](Theorem 1$)$, that it is also the connecting component of $\Gamma\left(\bmod \Lambda^{\prime}\right)$ (in particular, it can be embedded in a quiver of the form $\mathbb{Z} Q$, for some quiver $Q$ ). The conclusion then follows from the observation that, if $\Gamma^{\mathcal{B}}$ is non-semiregular, then, by [27](Theorem 2), $\Lambda^{\prime}$ is a laura algebra.

Again in contrast with the case of laura algebras, the component $\Gamma^{\mathcal{B}}$ is generally not faithful. For an example, we refer to Section 7.

\section{Laura Algebras and Trisections}

5.1. In this section, we are interested in a particular trisection of the module category. Let $\Lambda$ be an artin algebra, we let $E$ be the direct sum of a complet set of representatives of the Ext-injectives in $\operatorname{add}\left(\mathcal{L}_{\Lambda} \backslash \mathcal{R}_{\Lambda}\right)$, and $U$ be the direct sum of a complete set of representatives of the Ext-projectives in add $\left(\mathcal{R}_{\Lambda} \backslash \mathcal{L}_{\Lambda}\right)$. We set

$$
\begin{gathered}
\mathcal{L}_{\Lambda}^{\prime}=\left(\mathcal{L}_{\Lambda} \backslash \mathcal{R}_{\Lambda}\right) \backslash \text { ind } E, \\
\mathcal{R}_{\Lambda}^{\prime}=\left(\mathcal{R}_{\Lambda} \backslash \mathcal{L}_{\Lambda}\right) \backslash \text { ind } U, \\
\mathcal{B}=\operatorname{ind} \Lambda \backslash\left(\mathcal{L}_{\Lambda}^{\prime} \cup \mathcal{R}_{\Lambda}^{\prime}\right) .
\end{gathered}
$$

Lemma. The triple $\left(\mathcal{L}_{\Lambda}^{\prime}, \mathcal{B}, \mathcal{R}_{\Lambda}^{\prime}\right)$ is a trisection in ind $\Lambda$.

Proof. Clearly, $\mathcal{L}_{\Lambda} \backslash \mathcal{R}_{\Lambda}$ is closed under predecessors. Let $Y \in \mathcal{L}_{\Lambda}^{\prime}$ and $X \leadsto Y$ be a path in ind $\Lambda$. Since $Y \in \mathcal{L}_{\Lambda} \backslash \mathcal{R}_{\Lambda}$, then $X \in \mathcal{L}_{\Lambda} \backslash \mathcal{R}_{\Lambda}$. Assume thus that $X \in \operatorname{ind} E$. Since $Y$ belongs to $\mathcal{L}_{\Lambda} \backslash \mathcal{R}_{\Lambda}$, we have $Y \in \operatorname{ind} E$ as well, because of [1](6.3). This contradiction shows that $X \notin \operatorname{ind} E$, that is, $X \in \mathcal{L}_{\Lambda}^{\prime}$. Similarly, $\mathcal{R}_{\Lambda}^{\prime}$ is closed under successors. The statement now follows easily.

5.2. Lemma. Let $\Lambda$ be a laura algebra, then the trisection $\left(\mathcal{L}_{\Lambda}^{\prime}, \mathcal{B}, \mathcal{R}_{\Lambda}^{\prime}\right)$ is separated. If moreover $\Lambda$ is strict, then $\mathcal{B}$ is finite.

Proof. If $\Lambda$ is quasi-tilted, then the trisection $\left(\mathcal{L}_{\Lambda}^{\prime}, \mathcal{B}, \mathcal{R}_{\Lambda}^{\prime}\right)$ is clearly separated. We may thus assume that $\Lambda$ is strict laura. In this case, it follows from [3](4.6) that $\mathcal{B}$ is finite and that $\operatorname{add} \mathcal{L}_{\Lambda}^{\prime}$ is contravariantly finite while add $\mathcal{R}_{\Lambda}^{\prime}$ is covariantly finite. Let $f: X \rightarrow Y$ be a non-zero morphism with $X \in \mathcal{L}_{\Lambda}^{\prime}$ and $Y \in \mathcal{R}_{\Lambda}^{\prime}$. By [1](8.4), there exists $E_{1} \in \operatorname{add} E$ such that $f$ factors through $E_{1}$. Since $E_{1} \in$ $\operatorname{add} \mathcal{B}$, then the trisection is separated. 
5.3. We say that a path in ind $\Lambda$ is finite provided every morphism in this path belongs to a finite power of the radical of $\bmod \Lambda$.

Lemma. Let $\Lambda$ be an artin algebra, and $\mathcal{B}$ be an almost directed full convex subcategory of ind $\Lambda$. Then any path in $\mathcal{B}$ is finite.

Proof. Let $X=X_{0} \stackrel{f_{1}}{\rightarrow} X_{1} \rightarrow \cdots \stackrel{f_{t}}{\rightarrow} X_{t}=Y$ be a path in $\mathcal{B}$. If it is not finite, then there exists $i$ with $1 \leq i \leq t$ such that $f_{i} \in \operatorname{rad}_{\Lambda}^{\infty}\left(X_{i-1}, X_{i}\right)$. Then, by $[14](4.2 .2)$, there exist infinitely many non-isomorphic non-directed modules lying on a path from $X_{i-1}$ to $X_{i}$. Since $\mathcal{B}$ is convex in ind $\Lambda$, all these modules lie in $\mathcal{B}$, contradicting the hypothesis that $\mathcal{B}$ is almost directed.

5.4. Before stating, and proving, our main result of this section, we recall from [3] (2.4) that an algebra $\Lambda$ is laura if and only if any path in ind $\Lambda$ from an indecomposable projective to an indecomposable injective is finite.

THEOREM. Let $\Lambda$ be a representation-infinite artin algebra. The following conditions are equivalent.

(a) $\Lambda$ is a strict laura algebra, or a tilted algebra with a non-semiregular connecting component.

(b) $\left(\mathcal{L}_{\Lambda}^{\prime}, \mathcal{B}, \mathcal{R}_{\Lambda}^{\prime}\right)$ is a compact trisection.

(c) $\left(\mathcal{L}_{\Lambda}^{\prime}, \mathcal{B}, \mathcal{R}_{\Lambda}^{\prime}\right)$ is a separated trisection with addB functorially finite and $\mathcal{B}$ almost directed.

Proof. (a) $\Rightarrow$ (b). Assume that $\Lambda$ is strict laura. By $(5.2),\left(\mathcal{L}_{\Lambda}^{\prime}, \mathcal{B}, \mathcal{R}_{\Lambda}^{\prime}\right)$ is a separated trisection with $\mathcal{B}$ finite. Since $\Lambda$ is representation-infinite, then the trisection is compact. The proof is similar if $\Lambda$ is tilted with a non-semiregular connecting component.

(b) $\Rightarrow(\mathrm{c})$ This is trivial.

(c) $\Rightarrow$ (a). Assume that $\left(\mathcal{L}_{\Lambda}^{\prime}, \mathcal{B}, \mathcal{R}_{\Lambda}^{\prime}\right)$ satisfies the stated conditions. If all indecomposable injective modules lie in $\mathcal{R}_{\Lambda}$, or if all indecomposable projective modules lie in $\mathcal{L}_{\Lambda}$, then $\Lambda$ is quasi-tilted, by [13](II-1.14). Since $\mathcal{B}$ is almost directed, it is actually tilted and we are done. By [27](3.8), its connecting component is non-semiregular.

We may thus assume that $\Lambda$ is not quasi-tilted. Let $(*): I \leadsto P$ be a path in ind $\Lambda$ from an injective $I$ to a projective $P$. We prove now that $(*)$ is finite. If $I, P \in \mathcal{B}$, then this follows from (5.3). We may thus suppose that $I$ or $P$ does not lie in $\mathcal{B}$.

We claim that, if $I \in \mathcal{L}_{\Lambda}^{\prime}$, then any path in ind $\Lambda$ from $I$ to an indecomposable not in $\mathcal{R}_{\Lambda}^{\prime}$ is finite. Indeed, let

$$
I=X_{0} \stackrel{f_{1}}{\longrightarrow} X_{1} \longrightarrow \cdots \stackrel{f_{t}}{\longrightarrow} X_{t}=Y
$$

be a path in ind $\Lambda$ with $Y \notin \mathcal{R}_{\Lambda}^{\prime}$. Using that $\mathcal{L}_{\Lambda}^{\prime} \subset \mathcal{L}_{\Lambda}$ and it is closed under predecessors, it follows from [1](6.1) that $I$ has only finitely many successors 
in $\mathcal{L}_{\Lambda}^{\prime}$, all of which are Ext-injectives in $\mathcal{L}_{\Lambda}^{\prime}$. So the claim is proven if $Y \in$ $\mathcal{L}_{\Lambda}^{\prime}$. Suppose $Y \in \mathcal{B}$. Then there exists $i$ with $0 \leq i<t$ such that $X_{i} \in \mathcal{L}_{\Lambda}^{\prime}$ and $X_{i+1} \notin \mathcal{L}_{\Lambda}^{\prime}$. By [1](8.4), the morphism $f_{i}: X_{i} \rightarrow X_{i+1}$ factors through an indecomposable Ext-injective in $\mathcal{L}_{\Lambda}^{\prime}$. We may thus suppose that $X_{i}$ is Extinjective in $\mathcal{L}_{\Lambda}^{\prime}$. The morphism $f_{i}$ factors through the left minimal almost split morphism $h: X_{i} \rightarrow \bigoplus_{j} Z_{j}$. Clearly, we may assume that there exists $j_{0}$ such that $Z_{j_{0}} \notin \mathcal{L}_{\Lambda}^{\prime}$ and $\operatorname{Hom}_{\Lambda}\left(Z_{j_{0}}, X_{i+1}\right) \neq 0$. So we get a path

$$
I=X_{0} \leadsto X_{i} \stackrel{g}{\longrightarrow} Z_{j_{0}} \longrightarrow X_{i+1} \leadsto X_{t}=Y
$$

in ind $\Lambda$, with $g$ irreducible. The subpaths $X_{0} \leadsto X_{i}$ and $Z_{j_{0}} \leadsto Y$ are finite, by [1] (6.1) and (5.3), respectively (using that $Z_{j_{0}} \in \mathcal{B}$ by (2.2)). So the above path is finite. Since it is obtained by factoring $f_{i}$ through $h$, then the original path is finite. This establishes our claim.

Dually, if $P \in \mathcal{R}_{\Lambda}^{\prime}$, then any path from an indecomposable module not in $\mathcal{L}_{\Lambda}^{\prime}$ to $P$ is finite.

If $I \in \mathcal{L}_{\Lambda}^{\prime}$ and $P \notin \mathcal{R}_{\Lambda}^{\prime}$ or if $I \notin \mathcal{L}_{\Lambda}^{\prime}$ and $P \in \mathcal{R}_{\Lambda}^{\prime}$, then the required result follows from the claim and its dual. It remains to consider the case where $I \in \mathcal{L}_{\Lambda}^{\prime}$ and $P \in \mathcal{R}_{\Lambda}^{\prime}$. Since $\mathcal{B}$ is separating, we can assume the path $(*)$ to be of the form

$$
I=X_{0} \rightarrow \cdots \rightarrow X_{i-1} \rightarrow X_{i} \rightarrow \cdots \rightarrow X_{j} \rightarrow X_{j+1} \rightarrow \cdots \rightarrow X_{t}=P
$$

with $X_{i-1} \in \mathcal{L}_{\Lambda}^{\prime}, X_{i} \in \mathcal{B}, X_{j} \in \mathcal{B}$ and $X_{j+1} \in \mathcal{R}_{\Lambda}^{\prime}$. Applying (5.3) together with the claim and its dual, we get that $(*)$ is finite. Hence $\Lambda$ is a laura algebra. It is strict because we assumed it is not quasi-tilted.

5.5. We recall that an algebra $\Lambda$ is called strict weakly shod [12] if it is strict laura and its connecting component is directed.

COROLlary. Let $\Lambda$ be a representation-infinite artin algebra. The following statements are equivalent:

(a) $\Lambda$ is strict weakly shod or tilted with a non-semiregular connecting component.

(b) $\left(\mathcal{L}_{\Lambda}^{\prime}, \mathcal{B}, \mathcal{R}_{\Lambda}^{\prime}\right)$ is a compact trisection with $\mathcal{B}$ directed.

(c) $\left(\mathcal{L}_{\Lambda}^{\prime}, \mathcal{B}, \mathcal{R}_{\Lambda}^{\prime}\right)$ is a separated trisection with add $\mathcal{B}$ functorially finite and $\mathcal{B}$ directed.

\section{Laura Algebras And Cores}

6.1. Lemma. Let $\Lambda$ be an artin algebra, and $\Gamma$ be a component of $\Gamma(\bmod \Lambda)$.

(a) If $\Gamma$ contains an injective, then every module of $\Gamma \cap \mathcal{L}_{\Lambda}$ is directed.

(b) If $\Gamma$ contains a projective, then every module of $\Gamma \cap \mathcal{R}_{\Lambda}$ is directed.

Proof. This follows from [1](6.5). 
6.2. Let $\Lambda$ be an artin algebra. As before, we denote by $E$ (or by $U$ ) the direct sum of all indecomposable Ext-injectives in $\mathcal{L}_{\Lambda}$ (or Ext-projectives in $\mathcal{R}_{\Lambda}$, respectively). Also, we let $F$ stand for the direct sum of all indecomposable projectives not in $\mathcal{L}_{\Lambda}$ and $Q$ stand for the direct sum of all indecomposable injectives not in $\mathcal{R}_{\Lambda}$. Finally, $\Delta$ will denote the convex hull in ind $\Lambda$ of the modules in $\operatorname{ind}(E \oplus F \oplus U \oplus Q)$.

LEMMA. Let $\Lambda$ be a connected artin algebra such that

$$
\text { ind } \Lambda=(\text { PredE } \backslash \text { ind } E) \cup \Delta \cup(\text { Succ } U \backslash \text { ind } U) \text {. }
$$

(a) If $\mathcal{L}_{\Lambda} \neq \emptyset$, then $E \neq 0$ or $U \neq 0$.

(b) If $\mathcal{R}_{\Lambda} \neq \emptyset$, then $E \neq 0$ or $U \neq 0$.

Proof. We only prove (a), since the proof of (b) is dual.

(a) Assume $\mathcal{L}_{\Lambda} \neq \emptyset$, but $E=0$ and $U=0$. By hypothesis, ind $\Lambda=\Delta$, and $\Delta$ is the convex hull of $\operatorname{ind}(F \oplus Q)$. Assume $X \in \mathcal{L}_{\Lambda}$. Since $\mathcal{L}_{\Lambda} \subset \Delta$, there exist $X^{\prime}, X^{\prime \prime} \in \operatorname{ind}(F \oplus Q)$ such that we have paths $X^{\prime} \leadsto X \leadsto X^{\prime \prime}$. Suppose $X^{\prime} \in$ $\operatorname{ind} F$. Then $X^{\prime}$ is a projective not in $\mathcal{L}_{\Lambda}$. However $X \in \mathcal{L}_{\Lambda}$ which is closed under predecessors, hence a contradiction. Therefore, $X^{\prime} \in \operatorname{ind} Q$, that is, $X^{\prime}$ is an injective not in $\mathcal{R}_{\Lambda}$. However, $X \in \mathcal{L}_{\Lambda}$ implies $X^{\prime} \in \mathcal{L}_{\Lambda}$ and since $X^{\prime}$ is injective, we get $X^{\prime} \in \operatorname{ind} E$, by [1](6.1), a contradiction.

6.3. The main result of this section is the following theorem. Recall that an Ext-injective $M$ is of the first kind if there is an injective module $I$ and a path $I \leadsto M$. Otherwise it is called an Ext-injective of second kind (see [1] or [5]).

THEOREM. Let $\Lambda$ be a representation-infinite artin algebra which is not quasitilted. Then $\Lambda$ is strict laura if and only if

(a) $\mathcal{L}_{\Lambda} \neq \emptyset$ or $\mathcal{R}_{\Lambda} \neq \emptyset$.

(b) There is a component $\Gamma$ of $\Gamma(\bmod \Lambda)$ which contains a core $\Delta$ of $\Gamma$ and ind $\Lambda=(\operatorname{PredE} \backslash$ indE $) \cup \Delta \cup($ Succ $U \backslash$ ind $U)$.

Proof. Necessity. Since $\Lambda$ is laura and representation infinite, then (a) is satisfied. Let $\Gamma$ be the connecting component of $\Gamma(\bmod \Lambda)$. Then, since $\Lambda$ is strict, $Q \neq 0$ and $\operatorname{ind} Q \subset \Gamma$. Because $\Gamma$ is a convex component (see [27]), the convex hull $\Delta$ of $\operatorname{ind}(E \oplus F \oplus U \oplus Q)$ is contained in $\Gamma$. By [2], we have $\mathcal{L}_{\Lambda}=\operatorname{Pred} E$ and $\mathcal{R}_{\Lambda}=$ Succ $U$. Moreover, we can decompose $E$ in the form $E=E_{1} \oplus E_{2}$, where $E_{1}$ (or $E_{2}$ ) is the direct sum of the indecomposable Ext-injectives of the first kind (or of the second kind, respectively) and every indecomposable module not in $\mathcal{L}_{\Lambda}$ is generated by the tilting module $V=\tau_{\Lambda}^{-1} E_{2} \oplus E_{1} \oplus F$. Dually, every indecomposable module not in $\mathcal{R}_{\Lambda}$ is cogenerated by the cotilting module $W=\tau_{\Lambda} U_{2} \oplus U_{1} \oplus Q$, where $U_{1}$ (or $U_{2}$ ) is the direct sum of the indecomposable Ext-projectives of the first kind (or of the second kind, respectively).

Let $X \notin \mathcal{L}_{\Lambda} \cup \mathcal{R}_{\Lambda}$. Since $X \notin \mathcal{L}_{\Lambda}$, there exists an epimorphism $V^{m} \longrightarrow X$ (for some $m>0)$, and so $X \in \operatorname{Succ}(E \oplus F)$. Similarly, $X \notin \mathcal{R}_{\Lambda}$ gives a monomorphism 
$X \longrightarrow W^{n}$ (for some $n>0$ ), implying $X \in \operatorname{Pred}(U \oplus Q)$. This shows that $X$ lies in the convex hull $\Delta$ of $\operatorname{ind}(E \oplus F \oplus U \oplus Q)$.

We now want to show that $\Delta$ is a core. Since $\Delta \subset \Gamma$, and $\Gamma$ is quasi-directed, then $\Delta$ is almost directed. By definition, it is convex in ind $\Lambda$. Thus, there remains to show that $\Delta$ crosses each $\tau_{\Lambda}$-orbit of $\Gamma$ and only finitely many times. By [2], $E \neq 0$. Clearly, $\Gamma \cap$ ind $E \neq \emptyset$ and so, by [1](6.6), each $\tau_{\Lambda}$-orbit of $\Gamma \cap \mathcal{L}_{\Lambda}$ crosses ind $E$ exactly once. Dually, $U \neq 0$ and each $\tau_{\Lambda}$-orbit of $\Gamma \cap \mathcal{R}_{\Lambda}$ crosses ind $U$ exactly once. Suppose $X \notin \mathcal{L}_{\Lambda} \cup \mathcal{R}_{\Lambda}$. By the previous argument, $X \in \Delta$ and so the $\tau_{\Lambda}$-orbit $\mathcal{O}(X)$ of $X$ intersects $\Delta$. Therefore, $X \in \Gamma$ implies that $|\mathcal{O}(X) \cap \Delta| \geq 1$. We have to show that $|\mathcal{O}(X) \cap \Delta|<\infty$. Since $\Lambda$ is strict laura, then $\left|\mathcal{L}_{\Lambda} \cap \mathcal{R}_{\Lambda}\right|<\infty$ and $\left|\left(\mathcal{L}_{\Lambda} \cup \mathcal{R}_{\Lambda}\right)^{c}\right|<\infty$. If there exists $X \in \Gamma$ such that $|\mathcal{O}(X) \cap \Delta|=\infty$, then there exists in $\mathcal{O}(X)$ an infinity of non-isomorphic indecomposable modules $M_{i}$ in $\left(\mathcal{L}_{\Lambda} \backslash \mathcal{R}_{\Lambda}\right) \cap \Delta$ or in $\left(\mathcal{R}_{\Lambda} \backslash \mathcal{L}_{\Lambda}\right) \cap \Delta$ (here, we use the fact that

$$
\left[\left(\mathcal{L}_{\Lambda} \backslash \mathcal{R}_{\Lambda}\right) \cup\left(\mathcal{R}_{\Lambda} \backslash \mathcal{L}_{\Lambda}\right)\right]^{c}=\left(\mathcal{L}_{\Lambda} \cup \mathcal{R}_{\Lambda}\right)^{c} \cup\left(\mathcal{L}_{\Lambda} \cap \mathcal{R}_{\Lambda}\right)
$$

which is finite). Suppose the former case holds. Then an infinity of such modules belongs neither to ind $U$, nor to ind $Q$, nor to ind $F$ nor to ind $E$ (since the latter are finite). For each such $M_{i} \in \Delta$, we have a path $Y \leadsto M_{i} \leadsto Z$, with $Y, Z \in$ $\operatorname{ind}(E \oplus F \oplus U \oplus Q)$. Since $M_{i} \in \mathcal{L}_{\Lambda} \backslash \mathcal{R}_{\Lambda}$, we have $Y \in \operatorname{ind}(F \oplus U)$. Moreover, if $Y \in \operatorname{ind} E$, then infinitely many of the $M_{i}$ lie in ind $E$, an absurdity. Hence $Y \in \operatorname{ind} Q$, that is, $Y$ is injective. Since $Y \in \mathcal{L}_{\Lambda}$ (because $M_{i} \in \mathcal{L}_{\Lambda}$ ), then it is Ext-injective in $\mathcal{L}_{\Lambda}$ so $Y \in$ ind $E$ which is impossible as we just saw. This contradiction proves that $|\mathcal{O}(X) \cap \Delta|<\infty$ for $X \in \Gamma$. Therefore, $\Delta$ is a core. Finally, observe that $\operatorname{ind} \Lambda=(\operatorname{Pred} E \backslash \operatorname{ind} E) \cup \Delta \cup(\operatorname{Succ} U \backslash \operatorname{ind} U)$.

Sufficiency. In order to show that $\Lambda$ is laura, we show that the core $\Delta$ is finite. This suffices since Pred $E \subset \mathcal{L}_{\Lambda}$ and $\operatorname{Succ} U \subset \mathcal{R}_{\Lambda}$ (so that $\left(\mathcal{L}_{\Lambda} \cup \mathcal{R}_{\Lambda}\right)^{c} \subset \Delta$ ).

Since $\Lambda$ is not quasi-tilted, there exists an indecomposable injective module $I$ which is not in $\mathcal{R}_{\Lambda}$. Since $\operatorname{Succ} U \subset \mathcal{R}_{\Lambda}$ then $I \in \mathcal{L}_{\Lambda}$ or $I \in \Delta$. In both cases, $I \in \Delta$. Hence, $\Gamma$ contains an injective. By (6.1), $\mathcal{L}_{\Lambda} \cap \Gamma$ is directed. Similarly, $\mathcal{R}_{\Lambda} \cap \Gamma$ is directed. Since $\Delta$ is almost directed, we infer that $\Gamma$ is almost directed. We claim that $\Gamma$ is convex in ind $\Lambda$. Let

$$
X_{0} \stackrel{f_{1}}{\longrightarrow} X_{1} \longrightarrow \cdots \longrightarrow X_{t-1} \stackrel{f_{t}}{\longrightarrow} X_{t}
$$

be a path in ind $\Lambda$ with $X_{0}, X_{t} \in \Gamma$, and $X_{1}, \cdots, X_{t-1} \notin \Gamma$ (hence $t \geq 2$ ). Since $X_{0} \in \Gamma, X_{1} \notin \Gamma$, then $f_{1} \in \operatorname{rad}_{\Lambda}^{\infty}\left(X_{0}, X_{1}\right)$. Since, by [1](6.7), $\Gamma \cap \operatorname{ind} E$ is a left section of $\Gamma$, then there exist $E_{1} \in$ ind $E$ and a path

$$
X_{0}=X_{0}^{\prime} \stackrel{h_{1}}{\longrightarrow} X_{1}^{\prime} \longrightarrow \cdots \longrightarrow X_{s-1}^{\prime} \stackrel{h_{s}}{\longrightarrow} X_{s}^{\prime}=E_{1} \stackrel{g_{s}}{\longrightarrow} X_{1}
$$

with the $h_{i}$ irreducible, $g_{s} \in \operatorname{rad}_{\Lambda}^{\infty}\left(E_{1}, X_{1}\right)$ and $g_{s} h_{s} \cdots h_{1} \neq 0$. Dually, there exist $U_{1} \in \operatorname{ind} U$ and a path

$$
X_{t-1} \stackrel{l_{r}}{\longrightarrow} U_{1}=X_{0}^{\prime \prime} \stackrel{k_{1}}{\longrightarrow} X_{1}^{\prime \prime} \longrightarrow \cdots \longrightarrow X_{r-1}^{\prime} \stackrel{k_{r}}{\longrightarrow} X_{r}^{\prime \prime}=X_{t}
$$


with the $k_{i}$ irreducible, $l_{r} \in \operatorname{rad}_{\Lambda}^{\infty}\left(X_{t-1}, U_{1}\right)$ and $k_{r} \cdots k_{1} l_{r} \neq 0$. Since $E_{1}, U_{1} \in \Delta$, the convexity of $\Delta$ and the path

$$
E_{1} \longrightarrow X_{1} \longrightarrow X_{2} \longrightarrow \cdots \longrightarrow X_{t-1} \longrightarrow U_{1}
$$

yield $X_{1}, \cdots, X_{t-1} \in \Delta$, hence $X_{1}, \cdots, X_{t-1} \in \Gamma$, a contradiction which establishes our claim.

Applying [14](4.2.3), we get that $\Gamma$ is quasi-directed. In particular, $\Gamma$ has only finitely many $\tau_{\Lambda}$-orbits. Hence, so does $\Delta$. Since $\Delta$ is a core, then it intersects each $\tau_{\Lambda}$-orbit in $\Gamma$ finitely many times. Since $\Delta$ is almost directed, then it is finite. This finishes the proof of the theorem.

6.4. Corollary. Let $\Lambda$ be a representation-infinite artin algebra which is not quasi-tilted. Then $\Lambda$ is strict weakly shod if and only if

(a) $\mathcal{L}_{\Lambda} \neq \emptyset$ or $\mathcal{R}_{\Lambda} \neq \emptyset$.

(b) There exists a component $\Gamma$ of $\Gamma(\bmod \Lambda)$ which contains a directed core $\Delta$ such that ind $\Lambda=($ PredE $\backslash$ indE $) \cup \Delta \cup(S u c c U \backslash$ ind $U)$.

6.5. REMARK. If $\Lambda$ is representation-infinite and strict weakly hod, then the convex hull $\Delta$ of ind $(E \oplus F \oplus U \oplus Q)$ is equal to the convex hull $\Delta^{\prime}$ of ind $(E \oplus U)$. Indeed, since $\Lambda$ is strict weakly shod, then $\mathcal{L}_{\Lambda}=\operatorname{Pred} E$ and $\mathcal{R}_{\Lambda}=\operatorname{Succ} U$ by [2]. Let us show that $\left(\mathcal{L}_{\Lambda} \cup \mathcal{R}_{\Lambda}\right)^{c} \subset \Delta^{\prime}$. Let $X \notin \mathcal{L}_{\Lambda} \cup \mathcal{R}_{\Lambda}$ be indecomposable. By [21] (p.74), there exist an indecomposable $P_{1}$, an indecomposable injective $I_{1}$ and two non-sectional paths $I_{1} \leadsto X$ and $X \leadsto P_{1}$. Note first that, if $I_{1} \in \mathcal{L}_{\Lambda}$, then $I_{1} \in \operatorname{ind} E$. Under this assumption, suppose that $P_{1} \notin \mathcal{R}_{\Lambda}$ (otherwise, $X$ would belong to $\Delta^{\prime}$ ). Then there exist an indecomposable projective $P_{2}$ and a nonsectional path $P_{1} \leadsto P_{2}$. If $P_{2} \notin \mathcal{R}_{\Lambda}$, we get a longer path from $I_{1}$ to $P_{2}$. Since there are only finitely many projectives, and the lengths of paths from injectives to projectives are bounded (because $\Lambda$ is weakly shod), we get some $P_{s} \in \mathcal{R}_{\Lambda}$ hence $X \in \widetilde{\Delta}$.

This shows that $\operatorname{ind} \Lambda=(\operatorname{Pred} E \backslash \operatorname{ind} E) \cup \Delta^{\prime} \cup(\operatorname{Succ} U \backslash \operatorname{ind} U)$. Since $\Delta^{\prime} \subset \Delta$ and $\operatorname{ind} \Lambda=(\operatorname{Pred} E \backslash \operatorname{ind} E) \cup \Delta \cup(\operatorname{Succ} U \backslash \operatorname{ind} U)$, we get $\Delta^{\prime}=\Delta$.

\section{Quasi-laura Algebras and Further Generalisations}

7.1. We define a class of algebras which contains the laura algebras.

Definition. An algebra $\Lambda$ is quasi-laura if it admits a compact trisection $(\mathcal{A}, \mathcal{B}, \mathcal{C})$ such that the support algebras $A$ and $C$ of $\mathcal{A}, \mathcal{C}$, respectively, are quasitilted.

Observe that, by $[15,11]$, the only generalised standard component with sections of $A$, or $C$, are the postprojective, the preinjective and (eventually) the connecting component. The following is an example of a quasi-laura algebra which is not laura.

ExAMPLE. Let $\Lambda$ be given by the quiver 


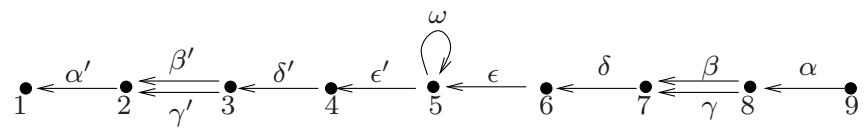

FiguRE 5.

bound by $\alpha \beta=0, \gamma \delta=0, \delta \epsilon=0, \epsilon \omega=0, \omega \epsilon^{\prime}=0, \epsilon^{\prime} \delta^{\prime}=0, \delta^{\prime} \gamma^{\prime}=0$ and $\beta^{\prime} \alpha^{\prime}=0$.

7.2. The results of the previous sections allow us to describe the AuslanderReiten components of a quasi-laura algebra $\Lambda$.

Corollary. Let $\Lambda$ be a quasi-laura algebra and $\Gamma$ be a component of $\Gamma(\bmod \Lambda)$. Then $\Gamma$ has one of the following shapes:

(a) It is the unique separating quasi-directed component, which is either nonsemiregular or has a section.

(b) It is a directed component, namely postprojective, preinjective or connecting component.

(c) It is a stable tube or a component of type $\mathbb{Z} A_{\infty}$.

(d) It is a component obtained from a stable tube or a component of type $\mathbb{Z} A_{\infty}$ by finitely many ray insertions and co-insertions.

Proof. This is a direct consequence of (4.2) and (4.4) using the well-known description of the Auslander-Reiten components of quasi-tilted algebras [16, 15, 11].

As can be easily seen in the above example, not only do we know the AuslanderReiten components of a quasi-laura algebra $\Lambda$, but also the existence of a trisection $(\mathcal{A}, \mathcal{B}, \mathcal{C})$ as in the definition yields the global structure of $\Gamma(\bmod \Lambda)$. Namely, inside each of the subcategories $\mathcal{A}$ or $\mathcal{C}$, morphisms behave as in their support algebras, which are quasi-tilted. Inside $\mathcal{B}$, which is a finite subcategory of ind $\Lambda$, morphisms behave as in a representation finite algebra. Also, morphims are from $\mathcal{A}$ to $\mathcal{B}$ and from $\mathcal{B}$ to $\mathcal{C}$ (and any morphism from $\mathcal{A}$ to $\mathcal{C}$ factors through add $\mathcal{B}$ ).

7.3. Definition. Let $m \geq 0$. An algebra $\Lambda$ is called $m$-quasi-laura provided

(a) If $m=0$, then $\Lambda$ is a quasi-tilted algebra.

(b) If $m \geq 1$, then $\Lambda$ admits a compact trisection $(\mathcal{A}, \mathcal{B}, \mathcal{C})$ such that the support algebra $A$ of $\mathcal{A}$ is a direct product of $n$-quasi-laura algebras, with $n<m$, at least one connected component of which is an $(m-1)$ quasi-laura algebra, and the support algebra $C$ of $\mathcal{C}$ is a direct product of quasi-tilted algebras.

7.4. EXAmples. (a) It follows directly from the definition that the 1-quasi-laura algebras are simply the quasi-laura algebras.

(b) The following is easily seen to be an example of a 2-quasi-laura algebra. Let $\Lambda$ be given by the quiver 


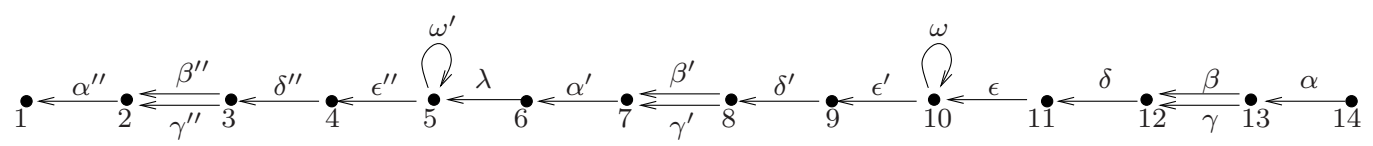

FiguRe 6.

bound by $\alpha \beta=0, \gamma \delta=0, \delta \epsilon=0, \epsilon \omega=0, \epsilon^{\prime} \delta^{\prime}=0, \delta^{\prime} \gamma^{\prime}=0, \beta^{\prime} \alpha^{\prime}=0, \alpha^{\prime} \lambda=$ $0, \lambda \omega^{\prime}=0, \omega^{\prime} \epsilon^{\prime \prime}=0, \epsilon^{\prime \prime} \delta^{\prime \prime}=0, \delta^{\prime \prime} \beta^{\prime \prime}=0, \beta^{\prime \prime} \alpha^{\prime \prime}=0$.

7.5. Corollary. Let $\Lambda$ be an m-quasi-laura algebra and $\Gamma$ be a component of $\Gamma(\bmod \Lambda)$. Then $\Gamma$ has one of the following shapes:

(a) It is a separating quasi-directed convex component, which is either nonsemiregular or has a section.

(b) It is a directed component, namely postprojective, preinjective or connecting component.

(c) It is a stable tube or a component of type $\mathbb{Z} A_{\infty}$.

(d) It is a component obtained from a stable tube or a component of type $\mathbb{Z} A_{\infty}$ by finitely many ray insertions and co-insertions.

Proof. This follows from (7.2) and an obvious induction.

7.6. Proposition. Let $\Lambda$ be an m-quasi-laura algebra. Then:

(a) there exists a separating quasi-directed convex component $\Gamma$ of $\Gamma(\bmod \Lambda)$ such that, if $\operatorname{Hom}_{\Lambda}\left(\Gamma^{\prime}, \Gamma\right) \neq 0$ and $\Gamma^{\prime} \neq \Gamma$, then $\Gamma^{\prime}$ is semiregular or regular.

(b) Let $(\mathcal{A}, \mathcal{B}, \mathcal{C})$ be a compact trisection of $\Lambda$ and $\Gamma=\Gamma^{\mathcal{B}}$. Then the support algebra $A$ of $\mathcal{A}$ is quasi-tilted, and the support algebra $C$ of $\mathcal{C}$ is $n$-quasilaura, with $n \leq m$.

Proof. We prove the statement by induction on $m$. If $m=0$, the statement is clear, because we can take $\Gamma$ to be a connecting component if $\Lambda$ is tilted or the preinjective component otherwise. Let $m \geq 1$ and assume that the result holds for all $n<m$. Because of (4.2), $\Lambda$ admits a compact trisection $\left(\mathcal{A}_{1}, \mathcal{B}_{1}, \mathcal{C}_{1}\right)$ such that $\Gamma(\bmod \Lambda)$ has a separating quasi-directed convex component $\Gamma^{\mathcal{B}_{1}}$, and all other components are components of $\Gamma\left(\bmod A_{1}\right)$ or $\Gamma\left(\bmod C_{1}\right)$, where $A_{1}\left(\right.$ or $\left.C_{1}\right)$ is the support algebra of $\mathcal{A}_{1}$ (or $\mathcal{C}_{1}$, respectively). Now $A_{1}$ has a connected component $A_{1}^{\prime}$ which is $(m-1)$-quasi-laura. By the induction hypothesis, $A_{1}^{\prime}$ admits a component $\Gamma$ verifying the required conditions in $(a)$. Now $\Gamma$ considered as a component of $\Gamma(\bmod \Lambda)$ also satisfies these conditions, because of the induction hypothesis and because for $\Gamma^{\mathcal{B}_{1}}$, and for any component $\Gamma^{\prime \prime}$ of $\Gamma\left(\bmod C_{1}\right)$, we have $\operatorname{Hom}_{\Lambda}\left(\Gamma^{\mathcal{B}_{1}}, \Gamma\right)=0, \operatorname{Hom}_{\Lambda}\left(\Gamma^{\prime \prime}, \Gamma\right)=0$. Moreover, let $(\mathcal{A}, \mathcal{B}, \mathcal{C})$ be a trisection such that $\Gamma=\Gamma^{\mathcal{B}}$, then the support algebra $A$ of $\mathcal{A}$ is quasi-tilted.

It seems reasonable that, if $\Lambda$ is $m$-quasi-laura, then so is $\Lambda^{o p}$. However, we do not know a proof of this fact. 
Acknowledgments. This paper was written when the first, the third and the fifth authors were visiting the Université de Sherbrooke under the partial support of NSERC, to whom they are grateful. The second author gratefully acknowledges partial support from the NSERC of Canada and the Université de Sherbrooke. The third author thanks the CNPq and FAPESP. The fifth author is a researcher of CONICET.

\section{REFERENCES}

1. I. Assem, Left sections and teh left part of an Artin algebra, Colloq. Math, to appear.

2. I. Assem, J. A. Cappa, M. I. Platzeck, S. Trepode, Some characterisations of supported algebras, J. Pure Appl. Algebra 208(3) (2007), 1121-1135.

3. I. Assem, F. U. Coelho, Two-sided gluings of tilted algebras, J. Algebra 269(2) (2003), 456-479.

4. I Assem, F. U. Coelho, M. Lanzilotta, D. Smith, S. Trepode, Algebras determined by their left and right parts, in Algebraic structures and their representations, volume 376 of Comtemp. Math. (2005) pages 13-47, Amer. Math. Soc., Providence, RI.

5. I. Assem, F. U. Coelho, S. Trepode, The left and the right parts of a module category, J. Algebra 281 (2004), 426-439.

6. I. Assem, F. U. Coelho, S. Trepode, Contravariantly finite subcategories closed under predecessors, preprint, 2008.

7. I. Assem, D. Simson, A. Skowroński, Elements of the representation theory of associative algebras, London Math. Soc. Students Texts 65, Cambridge University Press (2006).

8. M. Auslander, I. Reiten, S. Smalø, Representation Theory of Artin Algebras, volume 36 of Cambridge Studies in Advanced Mathematics. Cambridge University Press, 1995.

9. M. Auslander, S. Smalø, Preprojective modules over Artin algebras, J. Algebra 66 (1980), no. $1,61-122$.

10. M. Auslander, S. O. Smalø, Almost split sequences in subcategories, J. Algebra 69(2) (1981), 426-454. Addendum: J. Algebra 71(2), 592-594.

11. F. U. Coelho, Directing components for quasitilted algebras, Coll. Math. 82 (1999) 271-275.

12. F. U. Coelho, M. Lanzilotta, Weakly shod algebras, J. Algebra 265 (2003), 379-403.

13. D. Happel, I. Reiten, S. Smalø, Tilting in abelian categories and quasitilted algebras, Mem. Amer. Math. Soc. 120 (1996), no. 575, viii+ 88 pp.

14. M. Lanzilotta, D. Smith, Laura algebras and quasi-directed components, Colloq. Math. 105 (2006), 179-196.

15. H. Lenzing, A. Skowroński, Quasi-tilted algebras of canonical type, Colloq. Math. 71 (1996), no. 2, 161-181.

16. S. Liu, The connected components of the Auslander-Reiten quiver of a tilted algebra, J. Algebra 161 (2) (1993), 505-523.

17. J. A. de la Peña, Tame algebras with sincere directing modules, J. Algebra 161, no. 1 (1993), 171-185.

18. J. A. de la Peña, I. Reiten, Trisections of module categories, Colloq. Math. 107 (2007), no. 2, 191-219.

19. I. Reiten, A. Skowroński, Generalized double tilted algebras J. Math. Soc. Japan 56 (2004), no. 1, 269-288.

20. C. Ringel, Tame algebras and integral quadratic forms, Springer Lect. Notes Math. 1099 (1984).

21. C. M. Ringel, Report on the Brauer-Thrall conjecture, in Lecture Notes in Math. vol. 831, Springer-Verlag, Berlin, 1980, pp. 104-135. 
22. A. Skowroński, Minimal representation-infinite Artin algebras, Math. Proc. Cambridge Philos. Soc. 116 (1994), 229-243.

23. A. Skowroński, Generalised standard Auslander-Reiten components, J. Math. Soc. Japan 46 (1994), no. 3, 517-543.

24. A. Skowroński, Regular Auslander-Reiten components containing directing modules, Proc. Amer. Math. Soc. 120 (1994), no. 1, 19-26.

25. A. Skowroński, On artin algebras with almost all indecomposable of projective or injective dimension at most one, Cent. Eur. J. Math. 1(1) (2003), 108-122 (eletronic).

26. S. Smalø, Torsion theories and tilting modules, Bull. London Math. Soc. 16 (1984), no. 5, $518-522$.

27. D. Smith, On generalized standard Auslander-Reiten components having only finitely many non-directing modules, J. Algebra 279 (2004), 493-513.

Centro Politécnico, Departamento de Matemática, Universidade Federal do

Paraná, CP 019081, Jardim das AmÉricas, Curitiba-PR, 81531-990, Brazil

E-mail address: rolo@ufpr.br

Département de mathématiques, Université de Sherbrooke, Sherbrooke, Québec, CANADA, J1K 2R1.

E-mail address: ibrahim.assem@usherbrooke.ca

Departamento de Matemática-IMe, Universidade de São Paulo, CP 66281, São PAULO, SP, 05315-970, BRAZIL

E-mail address: fucoelho@ime.usp.br

Depto de Matemática, FCEyn, Universidad Nacional de Mar del Plata, 7600, Mar del Plata, Argentina

E-mail address: mapena@mdp.edu.ar

Depto de Matemática, FCEyn, Universidad Nacional de Mar del Plata, 7600, Mar del Plata, Argentina

E-mail address: strepode@mdp.edu.ar 